\title{
MUTUAL COHERENCE OF POLARIZED LIGHT IN DISORDERED MEDIA: TWO-FREQUENCY METHOD EXTENDED
}

\author{
ALBERT C. FANNJIANG
}

\begin{abstract}
The paper addresses the two-point correlations of electromagnetic waves in general random, bi-anisotropic media whose constitutive tensors are complex Hermitian, positive- or negative-definite matrices. A simplified version of the two-frequency Wigner distribution (2f-WD) for polarized waves is introduced and the closed form Wigner-Moyal equation is derived from the Maxwell equations. In the weak-disorder regime with an arbitrarily varying background the two-frequency radiative transfer (2f-RT) equations for the associated $2 \times 2$ coherence matrices are derived from the Wigner-Moyal equation by using the multiple scale expansion. In birefringent media, the coherence matrix becomes a scalar and the 2f-RT equations take the scalar form due to the absence of depolarization. A paraxial approximation is developed for spatialy anisotropic media. Examples of isotropic, chiral, uniaxial and gyrotropic media are discussed.
\end{abstract}

PACS numbers: 42.25.Dd, 41.20.Jb

\section{INTRODUCTION}

Consider the electromagnetic wave propagation in a random dielectric. Let $\mathbf{u}(\mathbf{x}, t)=$ $(\mathbf{D}(\mathbf{x}, t), \mathbf{B}(\mathbf{x}, t))^{\dagger}$ be the displacement-magnetic-induction vector field. Then the mutual coherence function is given by [3]

$$
\left\langle\mathbf{u}\left(\mathbf{x}_{1}, t_{1}\right) \mathbf{u}^{\dagger}\left(\mathbf{x}_{2}, t_{2}\right)\right\rangle=\int e^{i\left(\omega_{2}-\omega_{1}\right) t} e^{-i \tau\left(\omega_{1}+\omega_{2}\right) / 2}\left\langle\mathbf{U}\left(\mathbf{x}_{1}, \omega_{1}\right) \mathbf{U}^{\dagger}\left(\mathbf{x}_{2}, \omega_{2}\right)\right\rangle d \omega_{1} d \omega_{2}
$$

where $\langle\cdot\rangle$ is ensemble averaging, $t=\left(t_{1}+t_{2}\right) / 2, \tau=t_{1}-t_{2}$ and $\mathbf{U}(\mathbf{x}, \omega)$ is the frequency component of $\mathbf{u}$ at frequency $\omega$. Throughout, all vectors are by default column vectors and $\dagger$ denotes Hermitian conjugation.

Radiative transfer theory [1, 4, 8, 10, 13, 14, 15, 17] has been traditionally carried out in space-time with one time variable $\left(t_{1}=t_{2}\right)$. The main goal of this paper is to derive equations for the quantity directly related to $\left\langle\mathbf{U}\left(\mathbf{x}_{1}, \omega_{1}\right) \mathbf{U}^{\dagger}\left(\mathbf{x}_{2}, \omega_{2}\right)\right\rangle$. In particular, we obtain the twofrequency radiative transfer (2f-RT) equations for polarized light in the weak-disorder regime with an arbitrary bianisotropic background. The 2f-RT equations then determine, via (1), the two-space-time correlations of the electromagnetic wave in random media.

Our approach is set up for the most general linear local, lossless electromagnetic materials, in which each of the field vectors $\mathbf{E}$ and $\mathbf{H}$ is coupled tensorially to both $\mathbf{D}$ and $\mathbf{B}$. Such materials have been the subject of considerable recent interest. One reason is that they can be created as metamaterials, i.e. composites of more conventional materials in which $\mathbf{E}$ is coupled to $\mathbf{D}$ alone and $\mathbf{H}$ is coupled to $\mathbf{B}$ alone.

Department of Mathematics, University of California, Davis, CA 95616-8633. Email: fannjiang@math.ucdavis.edu The research is supported in part by the Defense Advanced Research Projects Agency (DARPA) grant N00014-02-1-0603 . 
The two-frequency approach has been previously pursued in terms of wavelength-rescaled two-frequency Wigner distribution (2f-WD) in the case of a uniform background [7]. In the present work, we introduce an alternative version of $2 \mathrm{f}-\mathrm{WD}$ and derive the corresponding 2 f-RT equations for the associated $2 \times 2$ coherence matrices in the case with an arbitrary background. We give several examples for which the scattering kernels can be computed explicitly. We show that birefringence naturally leads to decoupling of the polarization modes and the absence of depolarization in such media. As a result, the 2f-RT equations simplify to scalar equations.

In Section 2 we formulate the problem in terms of the straightforwardly defined $2 \mathrm{f}-\mathrm{WD}$ and derive the two-frequency Wigner-Moyal equation in Appendix A. In Section 3 we analyze the problem for high-frequency waves in an arbitrary background bianisotropic medium in the absence of random fluctuations. This is the geometrical optics regime. In Section 4 , we consider the weak-disorder regime where, in addition to the arbitrary background, small random fluctuations are present on the scale of the wavelength. In Section 5and 6 we employ the multiscale expansion technique to derive the radiative transfer equation from the twofrequency Wigner-Moyal equation for the weak-disorder regime. We also derive a paraxial approximation for the polarized light in a spatially anisotropic medium. In Section 7 we give several examples of isotropic, chiral, uniaxial and gyrotropic media for which the scattering kernels can be explicitly calculated. We conclude in Section 8 with a discussion of the final expression of the mutual coherence in terms of the solution of the $2 \mathrm{f}-\mathrm{RT}$ equations.

\section{Maxwell equations and Wigner-Moyal Equations}

In this paper, we consider the electromagnetic wave propagation in a heterogeneous, lossless, bi-anisotropic dielectric medium. We assume that the scattering medium is free of charges and currents and start with the source-free Maxwell equations in the frequency $\omega$ domain

$$
-i \omega\left[\begin{array}{l}
\mathbf{D} \\
\mathbf{B}
\end{array}\right]+\left[\begin{array}{cc}
0 & -\nabla \times \\
\nabla \times & 0
\end{array}\right] \mathbf{K}^{-1}\left[\begin{array}{l}
\mathbf{D} \\
\mathbf{B}
\end{array}\right]=0
$$

where $\mathbf{K}$ is, by the assumption of losslessness, a Hermitian matrix [12]

$$
\mathbf{K}=\left[\begin{array}{cc}
\mathbf{K}^{\epsilon} & \mathbf{K}^{\chi} \\
\mathbf{K}^{\chi \dagger} & \mathbf{K}^{\mu}
\end{array}\right]
$$

with the permittivity and permeability tensors $\mathbf{K}^{\epsilon}, \mathbf{K}^{\mu}$, and the magneto-electric tensor $\mathbf{K}^{\chi}$ [16]. The Hermitian matrix $\mathbf{K}$ is assumed to be invertible. The present formulation encompasses the acoustic, electromagnetic and elastic waves so that the $2 \mathrm{f}-\mathrm{RT}$ theory developed here can be extended to these waves without major changes. We choose $\mathbf{D}, \mathbf{B}$ as the primary fields because they are transverse (divergence-free).

In an isotropic dielectric, $\mathbf{K}^{\epsilon}=\epsilon \mathbf{I}, \mathbf{K}^{\mu}=\mu \mathbf{I}, \mathbf{K}^{\chi}=0$. In a biisotropic dielectric, $\mathbf{K}^{\chi}$ as well as $\mathbf{K}^{\epsilon}, \mathbf{K}^{\mu}$ are nonzero scalars. A reciprocal chiral medium is biisotropic with purely imaginary $\mathbf{K}^{\chi}=i \chi$. The appearance of nonzero $\mathbf{K}^{\chi}$ arises from the so called magnetoelectric effect [11]. Crystals are often naturally anisotropic, and in some media (such as liquid crystals) it is possible to induce anisotropy by applying e.g. an external electric field. In crystal optics, $\mathbf{K}^{\epsilon}, \mathbf{K}^{\mu}$ are real, symmetric matrices and $\mathbf{K}^{\chi}=0$ [3]. In response to a magnetic field, some materials can have a dielectric tensor that is complex-Hermitian; this is the gyrotropic effect. A magnetoelectric, bi-anisotropic medium has a constitutive relation 
(3) with complex Hermitian $\mathbf{K}^{\epsilon}, \mathbf{K}^{\mu}$ and a complex matrix $\mathbf{K}^{\chi}$ satisfying the Post constraint. It has been shown that a moving medium, even isotropic, must be treated as bi-anisotropic [5, 11.

Writing the total field $\mathbf{U}=(\mathbf{D}, \mathbf{B})$ we introduce the two-frequency matrix-valued Wigner distribution

$$
\mathbf{W}\left(\mathbf{x}, \mathbf{p} ; \omega_{1}, \omega_{2}\right)=\frac{1}{(2 \pi)^{3}} \int e^{-i \mathbf{p}^{\dagger} \mathbf{y}} \mathbf{U}_{1}\left(\mathbf{x}+\frac{\ell \mathbf{y}}{2}\right) \mathbf{U}_{2}^{\dagger}\left(\mathbf{x}-\frac{\ell \mathbf{y}}{2}\right) d \mathbf{y}
$$

where $\mathbf{U}_{1}$ and $\mathbf{U}_{2}$ are the total fields at frequencies $\omega_{1} / \ell$ and $\omega_{2} / \ell$ respectively. The parameter $\ell$ is roughly the ratio of the wavelength to the distance of propagation. In the present setting, $\ell \ll 1$. Correspondingly, we will replace $\omega$ in (2) by $\omega / \ell$. The $2 \mathrm{f}-\mathrm{WD}$ is clearly equivalent to the 2-point function $\mathbf{U}_{1} \mathbf{U}_{2}^{\dagger}$ via the inverse Fourier transform.

Notice the symmetry of the Wigner distribution matrix

$$
\mathbf{W}^{\dagger}\left(\mathbf{x}, \mathbf{p} ; \omega_{1}, \omega_{2}\right)=\mathbf{W}\left(\mathbf{x}, \mathbf{p} ; \omega_{2}, \omega_{1}\right) .
$$

In other words, the right hand side of (4) is invariant under the simultaneous transformations of Hermitian conjugation $\dagger$ and frequency exchange $\omega_{1} \leftrightarrow \omega_{2}$.

In what follows we shall omit writing the arguments of any fields unless necessary.

We put the equation (2) in the form of general symmetric hyperbolic system [17]

$$
-i \frac{\omega}{\ell} \mathbf{U}+\mathbf{R}_{j} \partial_{x_{j}}\left(\mathbf{K}^{-1} \mathbf{U}\right)=0
$$

where $\mathbf{R}_{j}$ are the symmetric matrix given by

$$
\mathbf{R}_{j}=\left[\begin{array}{cc}
0 & \mathbf{T}_{j} \\
-\mathbf{T}_{j} & 0
\end{array}\right]
$$

with

$$
\mathbf{T}_{1}=\left[\begin{array}{ccc}
0 & 0 & 0 \\
0 & 0 & -1 \\
0 & 1 & 0
\end{array}\right], \quad \mathbf{T}_{2}=\left[\begin{array}{ccc}
0 & 0 & 1 \\
0 & 0 & 0 \\
-1 & 0 & 0
\end{array}\right], \quad \mathbf{T}_{3}=\left[\begin{array}{ccc}
0 & -1 & 0 \\
1 & 0 & 0 \\
0 & 0 & 0
\end{array}\right]
$$

The matrices $i T_{j}, j=1,2,3$ are related to the photon spin matrices [2]. For ease of notation, we set $\mathbf{L}=\mathbf{K}^{-1}$.

Let $\omega^{\prime}=\left(\omega_{1}-\omega_{2}\right) / \ell$ and $\bar{\omega}=\left(\omega_{1}+\omega_{2}\right) / 2$. The 2f-WD satisfies the Wigner-Moyal equations

$$
\begin{aligned}
(7) i \omega^{\prime} \mathbf{W}= & \frac{i}{\ell} p_{j} \mathbf{R}_{j} \int e^{i \mathbf{q}^{\dagger} \mathbf{x}} \widehat{\mathbf{L}}(\mathbf{q}) \mathbf{W}\left(\mathbf{p}-\frac{\ell \mathbf{q}}{2}\right) d \mathbf{q}-\frac{i}{\ell} \int \mathbf{W}\left(\mathbf{p}+\frac{\ell \mathbf{q}}{2}\right) \widehat{\mathbf{L}}(\mathbf{q}) e^{i \mathbf{q}^{\dagger} \mathbf{x}} d \mathbf{q} p_{j} \mathbf{R}_{j} \\
& +\frac{1}{2} \mathbf{R}_{j} \partial_{x_{j}} \int e^{i \mathbf{q}^{\dagger} \mathbf{x}} \widehat{\mathbf{L}}(\mathbf{q}) \mathbf{W}\left(\mathbf{p}-\frac{\ell \mathbf{q}}{2}\right) d \mathbf{q}+\frac{1}{2} \partial_{x_{j}} \int \mathbf{W}\left(\mathbf{p}+\frac{\ell \mathbf{q}}{2}\right) \widehat{\mathbf{L}}(\mathbf{q}) e^{i \mathbf{q}^{\dagger} \mathbf{x}} d \mathbf{q} \mathbf{R}_{j} \\
(8) i \frac{2 \bar{\omega}}{\ell} \mathbf{W}= & \frac{i}{\ell} p_{j} \mathbf{R}_{j} \int e^{i \mathbf{q}^{\dagger} \mathbf{x}} \widehat{\mathbf{L}}(\mathbf{q}) \mathbf{W}\left(\mathbf{p}-\frac{\ell \mathbf{q}}{2}\right) d \mathbf{q}+\frac{i}{\ell} \int \mathbf{W}\left(\mathbf{p}+\frac{\ell \mathbf{q}}{2}\right) \widehat{\mathbf{L}}(\mathbf{q}) e^{i \mathbf{q}^{\dagger} \mathbf{x}} d \mathbf{q} p_{j} \mathbf{R}_{j} \\
& +\frac{1}{2} \mathbf{R}_{j} \partial_{x_{j}} \int e^{i \mathbf{q}^{\dagger} \mathbf{x}} \widehat{\mathbf{L}}(\mathbf{q}) \mathbf{W}\left(\mathbf{p}-\frac{\ell \mathbf{q}}{2}\right) d \mathbf{q}-\frac{1}{2} \partial_{x_{j}} \int \mathbf{W}\left(\mathbf{p}+\frac{\ell \mathbf{q}}{2} \widehat{\mathbf{L}}(\mathbf{q}) e^{i \mathbf{q}^{\dagger} \mathbf{x}} d \mathbf{q} \mathbf{R}_{j}\right.
\end{aligned}
$$

where $\widehat{\mathbf{L}}$ is the Fourier transform (spectral density) of $\mathbf{L}$

$$
\mathbf{L}(\mathbf{x})=\int e_{3}^{i \mathbf{x}^{\dagger} \mathbf{q}} \widehat{\mathbf{L}}(\mathbf{q}) d \mathbf{q} .
$$


The derivation is given in Appendix A. For a Hermitian $\mathbf{L}$ we have

$$
\hat{\mathbf{L}}(\mathbf{p})=\hat{\mathbf{L}}^{\dagger}(-\mathbf{p}), \quad \forall \mathbf{p} .
$$

Clearly eq. (7) is related to the time derivative of the mutual coherence (11) with respect to the central time $t$ while eq. (77) is related to the time derivative with respect to the differential time $\tau$. Our will focus first on eq. (7) and comment on the constraint posed by (8) in the Conclusion. The full analysis of eq. (8) requires substantially different treatment and will be presented elsewhere. However, we will discuss the constraint imposed by the leading order terms of eq. (8) in the Conclusion and its implication on the two-spacetime correlation.

\section{Geometrical optics}

In this regime, we let $\ell \ll 1$ implying a small ratio between the wavelength and the scale of background heterogeneity which is comparable to the distance of propagation.

Let us first simplify eq. (77) by expanding the expression in the power of $\ell$ and neglecting $O(\ell)$-terms. The first two terms on the right hand side of eq. (7) reduce to $\ell^{-1} \mathcal{P}_{0}-\mathcal{P}_{1}$ where

$$
\begin{aligned}
\mathcal{P}_{0}(\mathbf{p}) \mathbf{W} & =i p_{j} \mathbf{R}_{j} \mathbf{L W}-i \mathbf{W} \mathbf{L} p_{j} \mathbf{R}_{j} \\
\mathcal{P}_{1}(\mathbf{p}) \mathbf{W} & =\frac{1}{2} p_{j} \mathbf{R}_{j} \partial_{x_{l}} \mathbf{L} \partial_{p_{l}} \mathbf{W}+\frac{1}{2} \partial_{p_{l}} \mathbf{W} \partial_{x_{l}} \mathbf{L} p_{j} \mathbf{R}_{j}
\end{aligned}
$$

while the last two terms on the right hand side become

$$
\mathcal{P}_{2} \mathbf{W}=\frac{1}{2} \mathbf{R}_{j} \partial_{x_{j}}[\mathbf{L W}]+\frac{1}{2} \partial_{x_{j}}[\mathbf{W L}] \mathbf{R}_{j} .
$$

We employ the regular expansion $\mathbf{W}=\overline{\mathbf{W}}+\ell \mathbf{W}_{1}+\cdots$ and substitute it into the resulting equation. The leading-order equation

$$
\mathcal{P}_{0} \overline{\mathbf{W}}=0
$$

can be solved as follows [17].

For a positive (or negative) definite $\mathbf{L}, \mathbf{L}^{1 / 2}$ is well-defined and under the transformation $\mathbf{L}^{1 / 2}$ the matrix $p_{j} \mathbf{R}_{j} \mathbf{L}$ is transformed into the Hermitian matrix $\mathbf{L}^{1 / 2} p_{j} \mathbf{R}_{j} \mathbf{L}^{1 / 2}$ which has a complete set of eigenvectors and eigenvalues $\left\{\Omega^{\sigma}\right\} \subset \mathbb{R}$. Let $\left\{\mathbf{d}^{\sigma, \alpha}\right\}$ be the associated eigenvectors in the original vector space where the index $\alpha$ keeps track of the multiplicity. Let the eigenvectors $\left\{\mathbf{d}^{\sigma, \alpha}\right\}$ be normalized such that $\mathbf{d}^{\sigma, \alpha \dagger} \mathbf{L} \mathbf{d}^{\tau, \zeta}=\delta_{\sigma, \tau} \delta_{\alpha, \zeta}$. It is easy to check that $\left\{\mathbf{e}^{\sigma, \alpha \dagger}: \mathbf{e}^{\sigma, \alpha}=\mathbf{L d}^{\sigma, \alpha}\right\}$ are the left eigenvectors of $p_{j} \mathbf{R}_{j} \mathbf{L}$ and they are orthogonal to $\left\{\mathbf{d}^{\tau, \zeta}(\mathbf{p})\right\}$ with respect to the standard scalar product:

$$
\mathbf{e}^{\sigma, \alpha \dagger} \mathbf{d}^{\tau, \zeta}=\delta_{\sigma, \tau} \delta_{\alpha, \zeta} .
$$

Clearly, the eigenvalues $\Omega^{\sigma}$ as a function of the wavevector $\mathbf{p}$ define the dispersion relations. For general bianisotropic dielectric, it is easy to check that the zero eigenvalue $\Omega^{0}=0$ is always an eigenvalue with the associated left eigenvectors

$$
\mathbf{e}^{0,1}(\mathbf{p}) \sim\left(\begin{array}{c}
\mathbf{p} \\
0
\end{array}\right), \quad \mathbf{e}^{0,2}(\mathbf{p}) \sim\left(\begin{array}{l}
0 \\
\mathbf{p}
\end{array}\right) .
$$

Since $\mathbf{L}$ is invertible, it follows that the null space of $p_{j} \mathbf{R}_{j} \mathbf{L}$ is spanned by $\left\{\mathbf{d}^{0,1}=\mathbf{K e}^{0,1}, \mathbf{d}^{0,2}=\right.$ $\left.\mathbf{K e}^{0,2}\right\}$. The relations (10) and (11) imply that $\mathbf{d}^{\tau, \zeta}, \tau \neq 0$, are transverse vectors in the sense that they are orthogonal to the wavevector $\mathbf{p}$. 
Throughout the English indices represent the spatial degrees of freedom while the Greek indices represent the polarization degrees of freedom. The Einstein summation convention and the Hermitian conjugation are used only on the English indices.

Define

$$
\begin{aligned}
& \mathbf{D}^{\sigma, \alpha \zeta}(\mathbf{p}, \mathbf{q})=\mathbf{d}^{\sigma, \alpha}(\mathbf{p}) \mathbf{d}^{\sigma, \zeta}(\mathbf{q})^{\dagger} \\
& \mathbf{E}^{\sigma, \alpha \zeta}(\mathbf{p}, \mathbf{q})=\mathbf{e}^{\sigma, \alpha}(\mathbf{p}) \mathbf{e}^{\sigma, \zeta}(\mathbf{q})^{\dagger}
\end{aligned}
$$

The null space of $\mathcal{P}_{0}$ is the linear span of $\left\{\mathbf{D}^{\tau, \alpha \zeta}(\mathbf{p}, \mathbf{p}), \forall \tau, \alpha, \zeta, \mathbf{p}\right\}$, denoted by $\mathfrak{M}_{\mathbf{p}}$, for each $\mathbf{p} \neq 0$ with the scalar product $\operatorname{Tr}\left[\mathbf{H}^{\dagger} \mathbf{L G L}\right], \mathbf{H}, \mathbf{G} \in \mathfrak{M}_{\mathbf{p}}$.

Then the general solution to (9) can be expressed as

$$
\overline{\mathbf{W}}=\sum_{\sigma, \alpha, \zeta} \bar{W}_{\alpha \zeta}^{\sigma} \mathbf{D}^{\sigma, \alpha \zeta}(\mathbf{p}, \mathbf{p})
$$

where $\bar{W}_{\alpha \zeta}^{\sigma}$ are generally complex-valued functions. The matrices $\overline{\mathbf{W}}^{\sigma}=\left[\bar{W}_{\alpha \zeta}^{\sigma}\right]$, free of the English indices, are referred to as the coherence matrices.

The constraint that the electric displacement $\mathbf{D}$ and the magnetic induction $\mathbf{B}$ are both divergence-free yields

$$
( \pm \nabla, \pm \nabla) \cdot \overline{\mathbf{W}}=0
$$

which, in view of the definition (41), is equivalent to

$$
\left( \pm \mathbf{p}^{\dagger}, \pm \mathbf{p}^{\dagger}\right) \overline{\mathbf{W}}=0 .
$$

Hence by (111) $\mathbf{e}^{0, j \dagger} \overline{\mathbf{W}}=0$ and by (10) $\overline{\mathbf{W}}^{0}=0$ where $\mathbf{W}^{0}$ is the $2 \times 2$ coherence matrix associated with the non-propagating mode $\Omega^{0}=0$. This implies, by (10), that $\overline{\mathbf{W}}$ is a transverse field.

The $O(1)$ equation is

$$
\mathcal{P}_{0} \mathbf{W}_{1}=i \omega^{\prime} \overline{\mathbf{W}}+\mathcal{P}_{1} \overline{\mathbf{W}}-\mathcal{P}_{2} \overline{\mathbf{W}}
$$

which is solvable if the right hand side is orthogonal to the null space, $\mathfrak{M}_{\mathbf{p}}$, of $\mathcal{P}_{0}$. The solvability condition for (16) then leads to the governing equation for the $2 \times 2$ coherence matrices:

$$
i \omega^{\prime} \overline{\mathbf{W}}^{\tau}-\nabla_{\mathbf{x}} \Omega^{\tau} \cdot \nabla_{\mathbf{p}} \overline{\mathbf{W}}^{\tau}+\nabla_{\mathbf{p}} \Omega^{\tau} \cdot \nabla_{\mathbf{x}} \overline{\mathbf{W}}^{\tau}-\mathbf{C}^{\tau} \overline{\mathbf{W}}^{\tau}-\overline{\mathbf{W}}^{\tau} \mathbf{C}^{\tau \dagger}=0
$$

where the depolarization matrix $\mathbf{C}^{\tau}=\left[C_{\xi \alpha}^{\tau}\right]$ is given by

$$
C_{\xi \alpha}^{\tau}=\partial_{x_{j}} \Omega^{\tau} \mathbf{e}^{\tau, \xi \dagger} \partial_{p_{j}} \mathbf{d}^{\tau, \alpha}+\frac{1}{2}\left[\partial_{x_{j}} \mathbf{e}^{\tau, \xi \dagger} \mathbf{R}_{j} \mathbf{e}^{\tau, \alpha}-\mathbf{e}^{\tau, \xi \dagger} \mathbf{R}_{j} \partial_{x_{j}} \mathbf{e}^{\tau, \alpha}\right]
$$

Using (10) we can cast $\mathbf{C}^{\tau}$ in the explicitly skew-symmetric (in $\xi, \alpha$ ) form:

$$
C_{\xi \alpha}^{\tau}=\frac{1}{2} \partial_{x_{j}} \Omega^{\tau}\left[\mathbf{e}^{\tau, \xi \dagger} \mathbf{K} \partial_{p_{j}} \mathbf{e}^{\tau, \alpha}-\partial_{p_{j}} \mathbf{e}^{\tau, \xi \dagger} \mathbf{K} \mathbf{e}^{\tau, \alpha}\right]+\frac{1}{2}\left[\partial_{x_{j}} \mathbf{e}^{\tau, \xi \dagger} \mathbf{R}_{j} \mathbf{e}^{\tau, \alpha}-\mathbf{e}^{\tau, \xi \dagger} \mathbf{R}_{j} \partial_{x_{j}} \mathbf{e}^{\tau, \alpha}\right]
$$

The details of the calculation is given in Appendix B. Note that eq. (17) is invariant under the simultaneous transformations of Hermitian conjugation and frequency exchange. 


\section{WEAK-DISORDER REGIME}

Now we consider the weak coupling regime with the permittivity-permeability tensor $\mathbf{K}$ given by

$$
\mathbf{K}^{-1}(\mathbf{x})=\mathbf{L}_{0}(\mathbf{x})\left[\mathbf{I}+\sqrt{\ell} \mathbf{V}\left(\frac{\mathbf{x}}{\ell}\right)\right], \quad \ell \ll 1
$$

where the Hermitian matrix $\mathbf{L}_{0}$ represents the slowly varying background medium and $\sqrt{\ell} \mathbf{V}$ represents the medium fluctuations. The small parameter $\ell$ describes the ratio of the scale of the medium fluctuation or the wavelength to the propagation distance or the variability scale of $\mathbf{L}_{0}$.

To preserve the Hermicity of $\mathbf{L}$ the matrix $\mathbf{V}$ must satisfy

$$
\mathbf{V}^{\dagger} \mathbf{L}_{0}=\mathbf{L}_{0} \mathbf{V}
$$

We shall assume below that $\mathbf{L}_{0}$ is either positive or negative definite. A negative-definite $\mathbf{L}_{0}$ gives rise to negative index of refraction [18, 19]. A nondefinite $\mathbf{L}_{0}$ gives rise to complexvalued refractive index and hence a lossy medium. To fix the idea, let us take $\mathbf{L}_{0}$ to be positive definite. Our method applies equally well to the negative definite case.

We assume that $\mathbf{V}=\left[V_{i j}\right]$ is a stationary (statistically homogeneous) random field with the spectral density tensors $\boldsymbol{\Phi}=\left[\Phi_{i j m n}\right], \mathbf{\Psi}=\left[\Psi_{i j m n}\right]$ such that

$$
\begin{aligned}
\left\langle V_{i j}(\mathbf{x}) V_{m n}^{*}(\mathbf{y})\right\rangle & =\int e^{i \mathbf{k}^{\dagger}(\mathbf{x}-\mathbf{y})} \Phi_{i j m n}(\mathbf{k}) d \mathbf{k} \\
\left\langle V_{i j}(\mathbf{x}) V_{m n}(\mathbf{y})\right\rangle & =\int e^{i \mathbf{k}^{\dagger}(\mathbf{x}-\mathbf{y})} \Psi_{i j m n}(\mathbf{k}) d \mathbf{k}
\end{aligned}
$$

which implies

$$
\begin{aligned}
\left\langle\hat{V}_{i j}(\mathbf{p}) \hat{V}_{m n}^{*}(\mathbf{q})\right\rangle & =\Phi_{i j m n}(\mathbf{p}) \delta(\mathbf{p}-\mathbf{q}) \\
\left\langle\hat{V}_{i j}(\mathbf{p}) \hat{V}_{m n}(\mathbf{q})\right\rangle & =\Psi_{i j m n}(\mathbf{p}) \delta(\mathbf{p}+\mathbf{q}) .
\end{aligned}
$$

Here and below $*$ denotes the complex conjugation. In the case of real-valued $\mathbf{V}, \boldsymbol{\Phi}=\boldsymbol{\Psi}$. The spectral density tensors have the basic symmetry

$$
\begin{aligned}
\Phi_{i j m n}^{*}(\mathbf{p}) & =\Phi_{m n i j}(\mathbf{p}), \\
\Psi_{i j m n}(-\mathbf{p}) & =\Psi_{m n i j}(\mathbf{p}),
\end{aligned}
$$

Eq. (19) implies that

$$
\begin{aligned}
& L_{0, i j} \Psi_{m n j l}(\mathbf{p})=L_{0, l j}^{*} \Phi_{m n j i}(\mathbf{p}) \\
& L_{0, i j} \Phi_{m n j l}(\mathbf{p})={ }_{6}=L_{0, l j}^{*} \Psi_{m n j i}(\mathbf{p})
\end{aligned}
$$




$$
\begin{aligned}
i \omega^{\prime} \mathbf{W}= & \frac{i}{\ell} p_{j} \mathbf{R}_{j} \int e^{i \mathbf{q}^{\dagger} \mathbf{x}} \widehat{\mathbf{L}}_{0}(\mathbf{q}) \mathbf{W}\left(\mathbf{p}-\frac{\ell \mathbf{q}}{2}\right) d \mathbf{q}-\frac{i}{\ell} \int \mathbf{W}\left(\mathbf{p}+\frac{\ell \mathbf{q}}{2}\right) \widehat{\mathbf{L}}_{0}(\mathbf{q}) e^{i \mathbf{q}^{\dagger} \mathbf{x}} d \mathbf{q} p_{j} \mathbf{R}_{j} \\
& +\frac{1}{2} \mathbf{R}_{j} \partial_{x_{j}} \int e^{i \mathbf{q}^{\dagger} \mathbf{x}} \widehat{\mathbf{L}}_{0}(\mathbf{q}) \mathbf{W}\left(\mathbf{p}-\frac{\ell \mathbf{q}}{2}\right) d \mathbf{q}+\frac{1}{2} \partial_{x_{j}} \int \mathbf{W}\left(\mathbf{p}+\frac{\ell \mathbf{q}}{2}\right) \widehat{\mathbf{L}_{0}}(\mathbf{q}) e^{i \mathbf{q}^{\dagger} \mathbf{x}} d \mathbf{q} \mathbf{R}_{j} \\
& +\frac{i}{\sqrt{\ell}} p_{j} \mathbf{R}_{j} \int e^{i \mathbf{q}^{\dagger} \tilde{\mathbf{x}}} \widehat{\mathbf{L}_{0}}\left(\frac{\mathbf{q}^{\prime}}{\ell}\right) \widehat{\mathbf{V}}\left(\mathbf{q}-\mathbf{q}^{\prime}\right) \mathbf{W}\left(\mathbf{p}-\frac{\mathbf{q}}{2}\right) d \mathbf{q}^{\prime} d \mathbf{q} \\
& -\frac{i}{\sqrt{\ell}} \int \mathbf{W}\left(\mathbf{p}+\frac{\mathbf{q}}{2}\right) \widehat{\left(\mathbf{V}^{\dagger}\right)}\left(\mathbf{q}-\mathbf{q}^{\prime}\right) \widehat{\mathbf{L}_{0}}\left(\frac{\mathbf{q}^{\prime}}{\ell}\right) e^{i \mathbf{q}^{\dagger} \tilde{\mathbf{x}}} d \mathbf{q}^{\prime} d \mathbf{q} p_{j} \mathbf{R}_{j} \\
& +\frac{1}{2 \sqrt{\ell}} \mathbf{R}_{j} \partial_{\tilde{x}_{j}} \int e^{i \mathbf{q}^{\dagger} \tilde{\mathbf{x}}} \widehat{\mathbf{L}_{0}}\left(\frac{\mathbf{q}^{\prime}}{\ell}\right) \widehat{\mathbf{V}}\left(\mathbf{q}-\mathbf{q}^{\prime}\right) \mathbf{W}\left(\mathbf{p}-\frac{\mathbf{q}}{2}\right) d \mathbf{q}^{\prime} d \mathbf{q} \\
& +\frac{1}{2 \sqrt{\ell}} \partial_{\tilde{x}_{j}} \int \mathbf{W}\left(\mathbf{p}+\frac{\mathbf{q}}{2}\right) \widehat{\mathbf{V}^{\dagger}}\left(\mathbf{q}-\mathbf{q}^{\prime}\right) \widehat{\mathbf{L}_{0}}\left(\frac{\mathbf{q}^{\prime}}{\ell}\right) e^{i \mathbf{q}^{\dagger} \tilde{\mathbf{x}}} d \mathbf{q}^{\prime} d \mathbf{q} \mathbf{R}_{j}
\end{aligned}
$$

where $\tilde{\mathbf{x}}=\mathbf{x} / \ell$ is the fast spatial variable and $\widehat{\left(\mathbf{V}^{\dagger}\right)}(\mathbf{q})=\widehat{\mathbf{V}}^{\dagger}(-\mathbf{q})$ the Fourier transform of $\mathbf{V}^{\dagger}$. As in the geometrical optics we approximate the first four terms on the right hand side of eq. (28) by $\ell^{-1} \mathcal{P}_{0} \mathbf{W}-\mathcal{P}_{1} \mathbf{W}+\mathcal{P}_{2} \mathbf{W}$. For the last four terms on the right side of eq. (28) we have

$$
\begin{aligned}
\int e^{i \mathbf{q}^{\dagger} \tilde{\mathbf{x}}} \widehat{\mathbf{L}_{0}}\left(\frac{\mathbf{q}^{\prime}}{\ell}\right) \widehat{\mathbf{V}}\left(\mathbf{q}-\mathbf{q}^{\prime}\right) \mathbf{W}\left(\mathbf{p}-\frac{\mathbf{q}}{2}\right) d \mathbf{q}^{\prime} d \mathbf{q} \approx \mathbf{L}_{0}(\mathbf{x}) \int e^{i \mathbf{q}^{\dagger} \tilde{\mathbf{x}}} \widehat{\mathbf{V}}(\mathbf{q}) \mathbf{W}\left(\mathbf{p}-\frac{\mathbf{q}}{2}\right) d \mathbf{q} \\
\int \mathbf{W}\left(\mathbf{p}+\frac{\mathbf{q}}{2}\right) \widehat{\mathbf{V}^{\dagger}}\left(\mathbf{q}-\mathbf{q}^{\prime}\right) \widehat{\mathbf{L}_{0}}\left(\frac{\mathbf{q}^{\prime}}{\ell}\right) e^{i \mathbf{q}^{\dagger} \tilde{\mathbf{x}}} d \mathbf{q}^{\prime} d \mathbf{q} \approx \int \mathbf{W}\left(\mathbf{p}+\frac{\mathbf{q}}{2}\right) \widehat{\mathbf{V}^{\dagger}}(-\mathbf{q}) e^{i \mathbf{q}^{\dagger} \tilde{\mathbf{x}}} d \mathbf{q} \mathbf{L}_{0}(\mathbf{x})
\end{aligned}
$$

Hence we have the simplified form

$$
i \omega^{\prime} \mathbf{W}=\ell^{-1} \mathcal{P}_{0} \mathbf{W}-\mathcal{P}_{1} \mathbf{W}+\mathcal{P}_{2} \mathbf{W}+\ell^{-1 / 2} \mathcal{Q}_{1} \mathbf{W}+\ell^{-1 / 2} \mathcal{Q}_{2} \mathbf{W}
$$

where

$$
\begin{aligned}
\mathcal{Q}_{1} \mathbf{W} & =i p_{j} \mathbf{R}_{j} \mathbf{L}_{0} \int e^{i \mathbf{q}^{\dagger} \tilde{\mathbf{x}}} \widehat{\mathbf{V}}(\mathbf{q}) \mathbf{W}\left(\mathbf{p}-\frac{\mathbf{q}}{2}\right) d \mathbf{q}-i \int \mathbf{W}\left(\mathbf{p}+\frac{\mathbf{q}}{2}\right) \widehat{\mathbf{V}}^{\dagger}(\mathbf{q}) e^{i \mathbf{q}^{\dagger} \tilde{\mathbf{x}}} d \mathbf{q} \mathbf{L}_{0} p_{j} \mathbf{R}_{j} \\
\mathcal{Q}_{2} \mathbf{W} & =\frac{1}{2} \mathbf{R}_{j} \mathbf{L}_{0} \partial_{\tilde{x}_{j}} \int e^{i \mathbf{q}^{\dagger} \tilde{\mathbf{x}}} \widehat{\mathbf{V}}(\mathbf{q}) \mathbf{W}\left(\mathbf{p}-\frac{\mathbf{q}}{2}\right) d \mathbf{q}+\frac{1}{2} \partial_{\tilde{x}_{j}} \int \mathbf{W}\left(\mathbf{p}+\frac{\mathbf{q}}{2}\right) \widehat{\mathbf{V}}^{\dagger}(-\mathbf{q}) e^{i \mathbf{q}^{\dagger} \tilde{\mathbf{x}}} d \mathbf{q} \mathbf{L}_{0} \mathbf{R}_{j}
\end{aligned}
$$

Hereafter we shall work with eq. (29) to derive the 2f-RT equations by emplying the multiscale expansion (MSE).

\section{Multiscale expansion}

The key point of MSE is to separate the fast variable $\tilde{\mathbf{x}}$ from the slow variable $\mathbf{x}$ and make the substitution

$$
\nabla \mathbf{W} \rightarrow \nabla_{\mathbf{x}} \mathbf{W}+\ell^{-1} \nabla_{\tilde{\mathbf{x}}} \mathbf{W}
$$

Consequently,

$$
\mathcal{P}_{2} \mathbf{W} \rightarrow \mathcal{P}_{2} \mathbf{W}+\ell^{-1} \widetilde{\mathcal{P}}_{2} \mathbf{W}
$$


with

$$
\begin{aligned}
\mathcal{P}_{2} \mathbf{W} & =\frac{1}{2} \mathbf{R}_{j} \partial_{x_{j}}[\mathbf{L W}]+\frac{1}{2} \partial_{x_{j}}[\mathbf{W L}] \mathbf{R}_{j} \\
\widetilde{\mathcal{P}}_{2} \mathbf{W} & =\frac{1}{2} \mathbf{R}_{j} \mathbf{L} \partial_{\tilde{x}_{j}} \mathbf{W}+\frac{1}{2} \partial_{\tilde{x}_{j}} \mathbf{W} \mathbf{L} \mathbf{R}_{j} .
\end{aligned}
$$

The idea is that for sufficiently small $\ell$ the two widely separated scales, represented by $\mathbf{x}$ and $\tilde{\mathbf{x}}$ respectively, become mathematically independent.

We posit the expansion $\mathbf{W}=\overline{\mathbf{W}}+\sqrt{\ell} \mathbf{W}_{1}+\ell \mathbf{W}_{2}+\ldots$, substitute it into eq. (29) and equate terms of same order of magnitude.

The $O\left(\ell^{-1}\right)$ equation is

$$
\left(\widetilde{\mathcal{P}}_{2}+\mathcal{P}_{0}\right) \overline{\mathbf{W}}=0
$$

We hypothesize that the leading order term $\overline{\mathbf{W}}=\overline{\mathbf{W}}(\mathbf{x}, \mathbf{p})$ be independent of the fast variable $\tilde{\mathbf{x}}$. Thus $\widetilde{\mathcal{P}}_{2} \overline{\mathbf{W}}=0$ and eq. (32) reduces to (9) and its solution takes the form (14).

The $O\left(\ell^{-1 / 2}\right)$-equation is

$$
\mathcal{P}_{0} \mathbf{W}_{1}+\widetilde{\mathcal{P}}_{2} \mathbf{W}_{1}=-\mathcal{Q}_{1} \overline{\mathbf{W}}-\mathcal{Q}_{2} \overline{\mathbf{W}}
$$

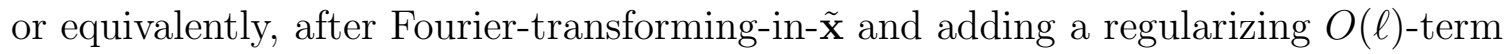

$$
\begin{aligned}
& -i 2 \ell \widehat{\mathbf{W}}_{1}(\mathbf{k}, \mathbf{p})+k_{j} \mathbf{R}_{j} \mathbf{L}_{0} \widehat{\mathbf{W}}_{1}(\mathbf{k}, \mathbf{p})+\widehat{\mathbf{W}}_{1}(\mathbf{k}, \mathbf{p}) \mathbf{L}_{0} k_{j} \mathbf{R}_{j} \\
& +2\left[p_{j} \mathbf{R}_{j} \mathbf{L}_{0} \widehat{\mathbf{W}}_{1}(\mathbf{k}, \mathbf{p})-\widehat{\mathbf{W}}_{1}(\mathbf{k}, \mathbf{p}) \mathbf{L}_{0} p_{j} \mathbf{R}_{j}\right] \\
& =-\mathbf{R}_{j} \mathbf{L}_{0}\left(2 p_{j}+k_{j}\right) \widehat{\mathbf{V}}(\mathbf{k}) \overline{\mathbf{W}}\left(\mathbf{p}-\frac{\mathbf{k}}{2}\right)+\overline{\mathbf{W}}\left(\mathbf{p}+\frac{\mathbf{k}}{2}\right) \widehat{\mathbf{V}}^{\dagger}(-\mathbf{k})\left(2 p_{j}-k_{j}\right) \mathbf{L}_{0} \mathbf{R}_{j}
\end{aligned}
$$

and posit the solution

$$
\widehat{\mathbf{W}}_{1}(\mathbf{k}, \mathbf{p})=\sum_{\sigma, \alpha, \zeta} C_{\alpha \zeta}^{\sigma}(\mathbf{k}, \mathbf{p}) \mathbf{D}^{\sigma, \alpha \zeta}\left(\mathbf{p}+\frac{\mathbf{k}}{2}, \mathbf{p}-\frac{\mathbf{k}}{2}\right)
$$

where $C_{\alpha \zeta}^{\sigma}$ are generally complex numbers. Note that the two arguments of $\mathbf{D}^{\sigma, \alpha \zeta}$ in (35) are at different momenta $\mathbf{p}+\mathbf{k} / 2, \mathbf{p}-\mathbf{k} / 2$.

We substitute (14) and (35) into eq. (34) and multiply it with $\mathbf{e}^{\sigma, \alpha}\left(\mathbf{p}+\frac{\mathbf{k}}{2}\right)^{\dagger}$ from the left and with $\mathbf{e}^{\sigma, \zeta}\left(\mathbf{p}-\frac{\mathbf{k}}{2}\right)$ from the right and solve the resulting equation algebraically. This yields the coefficients

$$
\begin{aligned}
C_{\alpha \zeta}^{\sigma}(\mathbf{k}, \mathbf{p})= & \left(\Omega^{\sigma}\left(\mathbf{p}+\frac{\mathbf{k}}{2}\right)-\Omega^{\sigma}\left(\mathbf{p}-\frac{\mathbf{k}}{2}\right)-i \ell\right)^{-1} \sum_{\eta} \\
& {\left[-\Omega^{\sigma}\left(\mathbf{p}+\frac{\mathbf{k}}{2}\right) \bar{W}_{\eta \zeta}^{\sigma}\left(\mathbf{p}-\frac{\mathbf{k}}{2}\right) \mathbf{e}^{\sigma, \alpha}\left(\mathbf{p}+\frac{\mathbf{k}}{2}\right)^{\dagger} \widehat{\mathbf{V}}(\mathbf{k}) \mathbf{d}^{\sigma, \eta}\left(\mathbf{p}-\frac{\mathbf{k}}{2}\right)\right.} \\
& \left.+\Omega^{\sigma}\left(\mathbf{p}-\frac{\mathbf{k}}{2}\right) \bar{W}_{\alpha \eta}^{\sigma}\left(\mathbf{p}+\frac{\mathbf{k}}{2}\right) \mathbf{d}^{\sigma, \eta}\left(\mathbf{p}+\frac{\mathbf{k}}{2}\right)^{\dagger} \widehat{\mathbf{V}}^{\dagger}(-\mathbf{k}) \mathbf{e}^{\sigma, \zeta}\left(\mathbf{p}-\frac{\mathbf{k}}{2}\right)\right] .
\end{aligned}
$$

When the leading term $\overline{\mathbf{W}}$ is invariant under the simultaneous transformations of Hermitian conjugation $\dagger$ and frequency exchange $\omega_{1} \leftrightarrow \omega_{2}$, so is $\mathbf{W}_{1}$ which is equivalent to

$$
C_{\zeta \alpha}^{\sigma *}\left(-\mathbf{k}, \mathbf{p} ; \omega_{1}, \omega_{2}\right)=C_{\alpha \zeta}^{\sigma}\left(\mathbf{k}, \mathbf{p} ; \omega_{2}, \omega_{1}\right) .
$$


Finally the $O(1)$-terms yields the equation after regularization

$$
\ell \mathbf{W}_{2}+\frac{1}{2} \mathbf{R}_{j} \mathbf{L}_{0} \frac{\partial}{\partial \tilde{x}_{j}} \mathbf{W}_{2}+\frac{1}{2} \frac{\partial}{\partial \tilde{x}_{j}} \mathbf{W}_{2} \mathbf{L}_{0} \mathbf{R}_{j}+i p_{j} \mathbf{R}_{j} \mathbf{L}_{0} \mathbf{W}_{2}-i \mathbf{W}_{2} \mathbf{L}_{0} p_{j} \mathbf{R}_{j}=\mathbf{F}
$$

with

$$
\mathbf{F}=i \omega^{\prime} \overline{\mathbf{W}}+\mathcal{P}_{1} \overline{\mathbf{W}}-\mathcal{P}_{2} \overline{\mathbf{W}}-\mathcal{Q}_{1} \mathbf{W}_{1}-\mathcal{Q}_{2} \mathbf{W}_{1} .
$$

It suffices to note that in order for the resulting solution $\ell \mathbf{W}_{2}$ to vanish in the limit $\ell \rightarrow 0$, F must satisfy the solvability condition

$$
\lim _{\ell \rightarrow 0} \operatorname{Tr}\left\langle\mathbf{G}^{\dagger} \mathbf{L}_{0} \mathbf{F} \mathbf{L}_{0}\right\rangle=0
$$

for all random stationary matrices $\mathbf{G}$ satisfying eq. (32). This can be seen by transforming eq. (37) into $\operatorname{Tr}\left\langle\mathbf{G}^{\dagger} \mathbf{L}_{0}\left(\right.\right.$ (37) $\left.\mathbf{L}_{0}\right\rangle$ which by eq. (32) implies $2 \ell \operatorname{Tr}\left\langle\mathbf{G}^{\dagger} \mathbf{L}_{0} \mathbf{W}_{2} \mathbf{L}_{0}\right\rangle=\operatorname{Tr}\left\langle\mathbf{G}^{\dagger} \mathbf{L}_{0} \mathbf{F L}_{0}\right\rangle$ and hence (39).

Fortunately, we do not need to work with the full solvability condition (39)). It suffices to demand (39) to be fulfilled by all deterministic $\mathbf{G}$, independent of $\tilde{\mathbf{x}}$, such that

$$
p_{j} \mathbf{R}_{j} \mathbf{L}_{0} \mathbf{G}-\mathbf{G L}_{0} p_{j} \mathbf{R}_{j}=0 \text {. }
$$

In other words, as in (14), we consider only a subspace of the null space of eq. (32) and replace (39) by

$$
\lim _{\ell \rightarrow 0} \operatorname{Tr}\left(\mathbf{E}^{\tau, \xi \nu \dagger}(\mathbf{p}, \mathbf{p})\langle\mathbf{F}(\mathbf{x}, \tilde{\mathbf{x}}, \mathbf{p})\rangle\right)=0, \quad \forall \tau, \xi, \nu, \mathbf{x}, \tilde{\mathbf{x}}, \mathbf{p}
$$

where $\mathbf{E}^{\tau, \xi \nu}$ are defined in (13). As (29), (33) and (38) are invariant under the simultaneous transformations of Hermitian conjugation $\dagger$ and frequency exchange $\omega_{1} \leftrightarrow \omega_{2}$, therefore eq. (41) must also be invariant under the same transformations.

To summarize, we have constructed the asymptotic solution $\overline{\mathbf{W}}+\sqrt{\ell} \mathbf{W}_{1}+\ell \mathbf{W}_{2}$ which satisfies approximately the $2 \mathrm{f}$ Wigner-Moyal equation in the sense that the remainder vanishes in a suitable sense as $\ell \rightarrow 0$ [6, 7].

With (35)-(36) and (38), eq. (41) is an implicit form of 2f-RT equations that determines the leading order coherence matrix. Our next step is to write (41) explicitly in terms of explicit, physical quantities.

\section{2F-RT EQUATIONS}

As in the geometrical optics, the terms $i \omega^{\prime} \overline{\mathbf{W}}+\mathcal{P}_{1} \overline{\mathbf{W}}-\mathcal{P}_{2} \mathbf{\mathbf { W }}$ in the expression (38) yield the left hand side of (17) after the operation (41).

First note the key expression

$$
\begin{aligned}
& \left\langle\int d \mathbf{q} e^{i \mathbf{q} \dagger \tilde{\mathbf{x}}} \hat{\mathbf{V}}(\mathbf{q}) \mathbf{W}_{1}\left(\mathbf{p}-\frac{\mathbf{q}}{2}\right)\right\rangle_{s j} \\
= & \sum_{\sigma, \alpha, \zeta, \eta} \int d \mathbf{k}\left(\Omega^{\sigma}(\mathbf{p}+\mathbf{k})-\Omega^{\sigma}(\mathbf{p})-i \ell\right)^{-1} \\
& \times\left[-\Omega^{\sigma}(\mathbf{p}+\mathbf{k}) \bar{W}_{\eta \zeta}^{\sigma}(\mathbf{p}) e_{f}^{\sigma, \alpha *}(\mathbf{p}+\mathbf{k}) \Psi_{f g s i}(\mathbf{k}) d_{g}^{\sigma, \eta}(\mathbf{p}) D_{i j}^{\sigma, \alpha \zeta}(\mathbf{p}+\mathbf{k}, \mathbf{p})\right. \\
& \left.+\Omega^{\sigma}(\mathbf{p}) \bar{W}_{\alpha \eta}^{\sigma}(\mathbf{p}+\mathbf{k}) d_{g}^{\sigma, \eta *}(\mathbf{p}+\mathbf{k}) \Phi_{f g s i}^{*}(-\mathbf{k}) e_{f}^{\sigma, \zeta}(\mathbf{p}) D^{\sigma, \alpha \zeta}(\mathbf{p}+\mathbf{k}, \mathbf{p})\right] \\
9 &
\end{aligned}
$$


and

$$
\begin{aligned}
& \left\langle\int d \mathbf{q} e^{i \mathbf{q} \dagger \tilde{\mathbf{x}}} \mathbf{W}_{1}\left(\mathbf{p}+\frac{\mathbf{q}}{2}\right) \hat{\mathbf{V}}(-\mathbf{q})^{\dagger}\right\rangle_{s j} \\
= & \sum_{\sigma, \alpha, \zeta, \eta} \int d \mathbf{k}\left(\Omega^{\sigma}(\mathbf{p})-\Omega^{\sigma}(\mathbf{p}-\mathbf{k})-i \ell\right)^{-1} \\
& \times\left[-\Omega^{\sigma}(\mathbf{p}) \bar{W}_{\eta \zeta}^{\sigma}(\mathbf{p}-\mathbf{k}) e_{f}^{\sigma, \alpha}(\mathbf{p})^{*} \Phi_{f g j n}(\mathbf{k}) d_{g}^{\sigma, \eta}(\mathbf{p}-\mathbf{k}) D_{s n}^{\sigma, \alpha \zeta}(\mathbf{p}, \mathbf{p}-\mathbf{k})\right. \\
& \left.+\Omega^{\sigma}(\mathbf{p}-\mathbf{k}) \bar{W}_{\alpha \eta}^{\sigma}(\mathbf{p}) d_{g}^{\sigma, \eta *}(\mathbf{p}) \Psi_{f g j n}^{*}(-\mathbf{k}) e_{f}^{\sigma, \zeta}(\mathbf{p}-\mathbf{k}) D_{s n}^{\sigma, \alpha \zeta}(\mathbf{p}, \mathbf{p}-\mathbf{k})\right] .
\end{aligned}
$$

The above expressions are independent of the fast variable $\tilde{\mathbf{x}}$ so $\left\langle\mathcal{Q}_{2} \mathbf{W}_{1}\right\rangle=0$. We also have

$$
\begin{aligned}
& \operatorname{Tr}\left(\mathbf{E}^{\sigma, \xi \nu \dagger}(\mathbf{p}, \mathbf{p})\left\langle\mathcal{Q}_{1} \mathbf{W}_{1}\right\rangle\right) \\
= & i \sum_{\alpha, \eta} \int d \mathbf{k}\left(\Omega^{\tau}(\mathbf{p}+\mathbf{k})-\Omega^{\tau}(\mathbf{p})-i \ell\right)^{-1} \Omega^{\sigma}(\mathbf{p}) e_{s}^{\sigma, \xi *}(\mathbf{p}) \\
& \times\left[-\Omega^{\sigma}(\mathbf{p}+\mathbf{k}) \bar{W}_{\eta \nu}^{\sigma}(\mathbf{p}) e_{f}^{\sigma, \alpha *}(\mathbf{p}+\mathbf{k}) \Psi_{f g s i}(\mathbf{k}) d_{g}^{\sigma, \eta}(\mathbf{p}) d_{i}^{\sigma, \alpha}(\mathbf{p}+\mathbf{k})\right. \\
+ & \left.\Omega^{\sigma}(\mathbf{p}) \bar{W}_{\alpha \eta}^{\sigma}(\mathbf{p}+\mathbf{k}) d_{g}^{\sigma, \eta *}(\mathbf{p}+\mathbf{k}) \Phi_{f g s i}^{*}(-\mathbf{k}) e_{f}^{\sigma, \nu}(\mathbf{p}) d_{i}^{\sigma, \alpha}(\mathbf{p}+\mathbf{k})\right] \\
& -i \sum_{\zeta, \eta} \int d \mathbf{k}\left(\Omega^{\sigma}(\mathbf{p})-\Omega^{\sigma}(\mathbf{p}-\mathbf{k})-i \ell\right)^{-1} \Omega^{\sigma}(\mathbf{p}) e_{j}^{\sigma, \nu}(\mathbf{p}) \\
& \times\left[-\Omega^{\sigma}(\mathbf{p}) \bar{W}_{\eta \zeta}^{\sigma}(\mathbf{p}-\mathbf{k}) e_{f}^{\sigma, \xi *}(\mathbf{p}) \Phi_{f g j n}(\mathbf{k}) d_{g}^{\sigma, \eta}(\mathbf{p}-\mathbf{k}) d_{n}^{\sigma, \zeta *}(\mathbf{p}-\mathbf{k})\right. \\
+ & \left.\Omega^{\sigma}(\mathbf{p}-\mathbf{k}) \bar{W}_{\xi \eta}^{\sigma}(\mathbf{p}) d_{g}^{\sigma, \eta *}(\mathbf{p}) \Psi_{f g j n}^{*}(-\mathbf{k}) e_{f}^{\sigma, \zeta}(\mathbf{p}-\mathbf{k}) d_{n}^{\sigma, \zeta *}(\mathbf{p}-\mathbf{k})\right]
\end{aligned}
$$

To state the full result in a concise form, let us introduce the following quantities. Define the scattering kernel tensors $\mathfrak{S}^{\tau}(\mathbf{p}, \mathbf{q})=\left[\mathcal{S}_{\xi \nu \alpha \zeta}^{\tau}(\mathbf{p}, \mathbf{q})\right]$ as

$$
\mathcal{S}_{\xi \alpha \nu \zeta}^{\tau}(\mathbf{p}, \mathbf{q})=\Omega^{\tau}(\mathbf{p}) \Omega^{\tau}(\mathbf{q}) e_{s}^{\tau, \xi *}(\mathbf{p}) d_{i}^{\tau, \alpha}(\mathbf{q}) \Phi_{s i f g}(\mathbf{p}-\mathbf{q}) e_{f}^{\tau, \nu}(\mathbf{p}) d_{g}^{\tau, \zeta *}(\mathbf{q})
$$

Using (24)-(27) one can derive the alternative expressions in terms of $\boldsymbol{\Psi}$

$$
\begin{aligned}
\mathcal{S}_{\xi \alpha \nu \zeta}^{\tau}(\mathbf{p}, \mathbf{q}) & =\Omega^{\tau}(\mathbf{p}) \Omega^{\tau}(\mathbf{q}) e_{f}^{\tau, \nu}(\mathbf{p}) d_{g}^{\tau, \zeta *}(\mathbf{q}) \Psi_{f g s i}^{*}(\mathbf{q}-\mathbf{p}) e_{s}^{\tau, \alpha}(\mathbf{q}) d_{i}^{\tau, \xi *}(\mathbf{p}) \\
& =\Omega^{\tau}(\mathbf{p}) \Omega^{\tau}(\mathbf{q}) e_{s}^{\tau, \xi *}(\mathbf{p}) d_{i}^{\tau, \alpha}(\mathbf{q}) \Psi_{s i f g}(\mathbf{q}-\mathbf{p}) e_{f}^{\tau, \zeta *}(\mathbf{q}) d_{g}^{\tau, \nu}(\mathbf{p})
\end{aligned}
$$

and the properties

$$
\begin{aligned}
& \mathcal{S}_{\xi \alpha \nu \zeta}^{\tau}(\mathbf{p}, \mathbf{q})=\mathcal{S}_{\nu \zeta \xi \alpha}^{\tau *}(\mathbf{p}, \mathbf{q}) \\
& \mathcal{S}_{\xi \alpha \nu \zeta}^{\tau}(\mathbf{q}, \mathbf{p})=\mathcal{S}_{\zeta \nu \alpha \xi}^{\tau}(\mathbf{p}, \mathbf{q})
\end{aligned}
$$

For any $\mathfrak{M}_{\mathbf{p}}$-valued field $\mathbf{G}(\mathbf{p})$ define the $(\xi, \nu)$-component of the tensor $\mathfrak{S}^{\tau}(\mathbf{p}, \mathbf{q}): \mathbf{G}(\mathbf{q})$ as

$$
\left[\mathfrak{S}^{\tau}(\mathbf{p}, \mathbf{q}): \mathbf{G}(\mathbf{q})\right]_{\xi \nu}=\sum_{\alpha, \zeta} \mathcal{S}_{\xi \alpha \nu \zeta}^{\tau}(\mathbf{p}, \mathbf{q}) G_{\alpha \zeta}(\mathbf{q})
$$

Define the tensors $\boldsymbol{\Sigma}^{\tau}=\left[\Sigma_{\xi \nu}^{\tau}\right]$ analogous to the total scattering cross section as

$$
\boldsymbol{\Sigma}^{\tau}(\mathbf{p})=\left\{\int \delta\left(\Omega^{\tau}(\mathbf{p})-\Omega^{\tau}(\mathbf{q})\right)-i f_{10}\left(\Omega^{\tau}(\mathbf{p})-\Omega^{\tau}(\mathbf{q})\right)^{-1}\right\} \mathfrak{S}^{\tau}(\mathbf{p}, \mathbf{q}): \mathbf{I} d \mathbf{q} .
$$


The 2f-RT equations for the coherence matrix $\overline{\mathbf{W}}^{\tau}$ then reads as

$$
\begin{aligned}
& i \omega^{\prime} \overline{\mathbf{W}}^{\tau}-\nabla_{\mathbf{x}} \Omega^{\tau} \cdot \nabla_{\mathbf{p}} \overline{\mathbf{W}}^{\tau}+\nabla_{\mathbf{p}} \Omega^{\tau} \cdot \nabla_{\mathbf{x}} \overline{\mathbf{W}}^{\tau}-\mathbf{C}^{\tau} \overline{\mathbf{W}}^{\tau}-\overline{\mathbf{W}}^{\tau} \mathbf{C}^{\tau \dagger} \\
= & 2 \pi \int \delta\left(\Omega^{\tau}(\mathbf{q})-\Omega^{\tau}(\mathbf{p})\right) \mathfrak{S}^{\tau}(\mathbf{p}, \mathbf{q}): \overline{\mathbf{W}}^{\tau}(\mathbf{q}) d \mathbf{q}-\boldsymbol{\Sigma}^{\tau}(\mathbf{p}) \overline{\mathbf{W}}^{\tau}(\mathbf{p})-\overline{\mathbf{W}}^{\tau}(\mathbf{p}) \boldsymbol{\Sigma}^{\tau \dagger}(\mathbf{p}), \quad \forall \tau .
\end{aligned}
$$

The $\delta$-function and Cauchy singular kernel arise because of the fact

$$
\lim _{\ell \rightarrow 0} \frac{1}{x-i \ell}=i \pi \delta(x)+\frac{1}{x}
$$

in the sense of generalized function.

With the property (43) one can verify directly the invariance of (45) with respect to the simultaneous transformations of Hermitian conjugation and frequency exchange.

6.1. Birefringence: scalar 2f-RT equation. Although, in view of (11), the zero eigenvalue $\Omega^{0}=0$ has multiplicity two in general, the nonzero eigenvalues in media other than the simplest isotropic medium often have multiplicity one as we shall see in Section 7 . This is closely related to the birefringence effect. Under such circumstances, the 2f-RT equations take a much simplified form which we now state.

Because $\Omega^{j}, j=1,2,3,4$ are simple (of multiplicity one), expression (14) reduces to

$$
\overline{\mathbf{W}}=\sum_{\sigma} \bar{W}^{\sigma} \mathbf{D}^{\sigma}(\mathbf{p}, \mathbf{p}) .
$$

Consequently (45) becomes a scalar equation for $\bar{W}^{\sigma}$ and the different polarization modes decouple:

$$
\begin{aligned}
& i \omega^{\prime} \bar{W}^{\tau}-\nabla_{\mathbf{x}} \Omega^{\tau} \cdot \nabla_{\mathbf{p}} \bar{W}^{\tau}+\nabla_{\mathbf{p}} \Omega^{\tau} \cdot \nabla_{\mathbf{x}} \bar{W}^{\tau} \\
= & 2 \pi \int \delta\left(\Omega^{\tau}(\mathbf{q})-\Omega^{\tau}(\mathbf{p})\right) \mathfrak{S}^{\tau}(\mathbf{p}, \mathbf{q}) \bar{W}^{\tau}(\mathbf{q}) d \mathbf{q}-2 \Sigma^{\tau}(\mathbf{p}) \bar{W}^{\tau}(\mathbf{p}), \quad \forall \tau
\end{aligned}
$$

where

$$
\begin{aligned}
\mathfrak{S}^{\tau}(\mathbf{p}, \mathbf{q}) & =\Omega^{\tau}(\mathbf{p}) \Omega^{\tau}(\mathbf{q}) e_{s}^{\tau *}(\mathbf{p}) d_{i}^{\tau}(\mathbf{q}) \Phi_{s i f g}(\mathbf{p}-\mathbf{q}) e_{f}^{\tau}(\mathbf{p}) d_{g}^{\tau *}(\mathbf{q}) \\
\Sigma^{\tau}(\mathbf{p}) & =\pi \int \delta\left(\Omega^{\tau}(\mathbf{p})-\Omega^{\tau}(\mathbf{q})\right) \mathfrak{S}^{\tau}(\mathbf{p}, \mathbf{q}) d \mathbf{q}
\end{aligned}
$$

Note that the Cauchy principal value integral disappears from (49) whenever $\Sigma^{\tau}$ and $\overline{\mathbf{W}}$ commute.

6.2. Paraxial approximation: spatial anisotropy. Consider now a spatially anisotropic spectral density tensor for a medium fluctuating much more slowly in the longitudinal $x_{3}=z$ direction, i.e. replacing $\boldsymbol{\Phi}(\mathbf{p}-\mathbf{q})$ in (45) by

$$
\frac{1}{\theta} \boldsymbol{\Phi}\left(\mathbf{p}_{\perp}-\mathbf{q}_{\perp}, \frac{1}{\theta}(p-q)\right)
$$

which, in the limit $\theta \rightarrow 0$, tends to

$$
\delta(p-q) \int d k \boldsymbol{\Phi}\left(\mathbf{p}_{\perp}-\mathbf{q}_{\perp}, k\right) .
$$


With $\overline{\mathbf{W}}^{\sigma}=\overline{\mathbf{W}}^{\sigma}\left(\mathbf{x}_{\perp}, z, \mathbf{p}_{\perp}, p\right)$, the right hand side of eq. (45) reduces to

$$
\begin{aligned}
& 2 \pi \int \delta\left(\Omega^{\tau}\left(\mathbf{p}_{\perp}, p\right)-\Omega^{\tau}\left(\mathbf{q}_{\perp}, p\right)\right) \mathfrak{S}\left(\mathbf{p}_{\perp}, \mathbf{q}_{\perp}\right): \overline{\mathbf{W}}^{\tau}\left(\mathbf{q}_{\perp}\right) d \mathbf{q}_{\perp} \\
& -\left[\boldsymbol{\Sigma}^{\tau}\left(\mathbf{p}_{\perp}\right) \overline{\mathbf{W}}^{\tau}\left(\mathbf{p}_{\perp}\right)+\overline{\mathbf{W}}^{\tau}\left(\mathbf{p}_{\perp}\right) \boldsymbol{\Sigma}^{\tau \dagger}\left(\mathbf{p}_{\perp}\right)\right]
\end{aligned}
$$

where $\mathfrak{S}^{\tau}=\left[\mathcal{S}_{\xi \alpha \nu \zeta}^{\tau}\right]$

$$
\mathcal{S}_{\xi \alpha \nu \zeta}^{\tau}\left(\mathbf{p}_{\perp}, \mathbf{q}_{\perp}\right)=e_{s}^{\tau, \xi *}\left(\mathbf{p}_{\perp}, p\right) d_{i}^{\tau, \alpha}\left(\mathbf{q}_{\perp}, p\right) \int \Phi_{s i f g}\left(\mathbf{p}_{\perp}-\mathbf{q}_{\perp}, k\right) d k e_{f}^{\tau, \nu}\left(\mathbf{p}_{\perp}, p\right) d_{g}^{\tau, \zeta *}\left(\mathbf{q}_{\perp}, p\right)
$$

and

$\boldsymbol{\Sigma}^{\tau}\left(\mathbf{p}_{\perp}\right)=\left[\int \delta\left(\Omega^{\tau}\left(\mathbf{p}_{\perp}, p\right)-\Omega^{\tau}\left(\mathbf{q}_{\perp}, p\right)\right)-i f\left(\Omega^{\tau}\left(\mathbf{p}_{\perp}, p\right)-\Omega^{\tau}\left(\mathbf{q}_{\perp}, p\right)\right)^{-1}\right] \mathfrak{S}^{\tau}\left(\mathbf{p}_{\perp}, \mathbf{q}_{\perp}\right): \mathbf{I} d \mathbf{q}_{\perp}$.

Eq. (45) now takes the paraxial form

$$
\begin{aligned}
& \partial_{p} \Omega^{\tau} \partial_{z} \mathfrak{W}^{\tau}+\nabla_{\mathbf{p}_{\perp}} \Omega^{\tau} \cdot \nabla_{\mathbf{x}_{\perp}} \overline{\mathbf{W}}^{\tau}+i \omega^{\prime} \overline{\mathbf{W}}^{\tau}-\nabla_{\mathbf{x}} \Omega^{\tau} \cdot \nabla_{\mathbf{p}} \overline{\mathbf{W}}^{\tau}-\mathbf{C}^{\tau} \overline{\mathbf{W}}^{\tau}-\overline{\mathbf{W}}^{\tau} \mathbf{C}^{\tau \dagger} \\
= & 2 \int \delta\left(\Omega^{\tau}\left(\mathbf{p}_{\perp}, p\right)-\Omega^{\tau}\left(\mathbf{q}_{\perp}, p\right)\right) \mathfrak{S}\left(\mathbf{p}_{\perp}, \mathbf{q}_{\perp}\right): \mathfrak{W}^{\tau}\left(\mathbf{q}_{\perp}\right) d \mathbf{q}_{\perp} \\
& -\left[\boldsymbol{\Sigma}^{\tau}\left(\mathbf{p}_{\perp}\right) \mathfrak{W}^{\tau}\left(\mathbf{p}_{\perp}\right)+\mathfrak{W}^{\tau}\left(\mathbf{p}_{\perp}\right) \boldsymbol{\Sigma}^{\tau \dagger}\left(\mathbf{p}_{\perp}\right)\right] .
\end{aligned}
$$

The longitudinal variable $z$ plays the role of a temporal variable and $p$ is a parameter so that (51) can be solved as an "initial" value problem given the initial data on $z=$ constant and a fixed $p$ if $\partial_{p} \Omega^{\tau} \neq 0$.

\section{EXAMPLES}

In this section, we briefly discuss a few media for which the scattering tensor can be explicitly computed (see [7] for a more elaborate discussion).

7.1. Isotropic medium. For the simplest isotropic medium, $\mathbf{K}_{0}=\operatorname{diag}\left[\epsilon_{0}, \epsilon_{0}, \epsilon_{0}, \mu_{0}, \mu_{0}, \mu_{0}\right]$. There are two nozero eigenvalues: $\Omega^{+}(\mathbf{p})=c_{0}|\mathbf{p}|, \Omega^{-}(\mathbf{p})=-c_{0}|\mathbf{p}|$ of multiplicity two. Let $\hat{\mathbf{p}}=\mathbf{p} /|\mathbf{p}|$ and let $\hat{\mathbf{p}}_{\perp}^{+}, \hat{\mathbf{p}}_{\perp}^{-}$be any pair of unit vectors orthogonal to each other and to $\hat{\mathbf{p}}$ so that $\left\{\hat{\mathbf{p}}_{\perp}^{+}, \hat{\mathbf{p}}_{\perp}^{-}, \hat{\mathbf{p}}\right\}$ form a right-handed coordinate frame. Let $\left\{\hat{\mathbf{q}}_{\perp}^{+}, \hat{\mathbf{q}}_{\perp}^{-}, \hat{\mathbf{q}}\right\}$ be similarly defined. The eigenvectors are

$\mathbf{d}^{+,+}(\mathbf{p})=\left(\begin{array}{c}\sqrt{\frac{\epsilon_{0}}{2}} \hat{\mathbf{p}}_{\perp}^{+} \\ \sqrt{\frac{\mu_{0}}{2}} \hat{\mathbf{p}}_{\perp}^{-}\end{array}\right), \mathbf{d}^{+,-}(\mathbf{p})=\left(\begin{array}{c}\sqrt{\frac{\epsilon_{0}}{2}} \hat{\mathbf{p}}_{\perp}^{-} \\ -\sqrt{\frac{\mu_{0}}{2}} \hat{\mathbf{p}}_{\perp}^{+}\end{array}\right), \mathbf{d}^{-,+}(\mathbf{p})=\left(\begin{array}{c}\sqrt{\frac{\epsilon_{0}}{2}} \hat{\mathbf{p}}_{\perp}^{+} \\ -\sqrt{\frac{\mu_{0}}{2}} \hat{\mathbf{p}}_{\perp}^{-}\end{array}\right), \mathbf{d}^{-,-}(\mathbf{p})=\left(\begin{array}{c}\sqrt{\frac{\epsilon_{0}}{2}} \hat{\mathbf{p}}_{\perp}^{-} \\ \sqrt{\frac{\mu_{0}}{2}} \hat{\mathbf{p}}_{\perp}^{+}\end{array}\right)$.

Often, in a scattering atmosphere for instance, $\tilde{\mu} \approx 0$ and consequently

$$
\mathcal{S}_{\xi \alpha \nu \zeta}^{\tau}(\mathbf{p}, \mathbf{q})=\frac{1}{4} \Phi_{\epsilon}(\mathbf{p}-\mathbf{q}) \hat{\mathbf{p}}_{\perp}^{\xi \dagger} \hat{\mathbf{q}}_{\perp}^{\alpha} \hat{\mathbf{q}}_{\perp}^{\zeta \dagger} \hat{\mathbf{p}}_{\perp}^{\nu}, \quad \tau, \xi, \alpha, \nu, \zeta= \pm .
$$

This is the setting for which S. Chandrasekhar originally derived his famous equation of transfer [4]. In this case, eq. (45) is the two-frequency version of Chandrasekhar's famous transfer equation [4, 9].

As we shall see below, many materials are birefringent and permit two monochromatic plane waves with two different linear polarizations and two different velocities to propagate in any given direction [3]. This is the birefringence effect. 
7.2. Chiral media. A chiral medium is a reciprocal, biisotropic medium with the constitutive matrix

$$
\mathbf{K}_{0}=\left[\begin{array}{cc}
\epsilon_{0} \mathbf{I} & i \chi \mathbf{I} \\
-i \chi \mathbf{I} & \mu_{0} \mathbf{I}
\end{array}\right]
$$

where $\chi \in \mathbb{R}$ is the magneto-electric coefficient. To maintain a positive-definite $\mathbf{K}_{0}$ we assume $\chi^{2}<\epsilon \mu$. We then have

$$
p_{j} \mathbf{R}_{j} \mathbf{L}_{0}=\frac{c_{0}}{1-\kappa^{2}}\left[\begin{array}{cc}
0 & -\mathbf{p} \times \\
\mathbf{p} \times & 0
\end{array}\right]\left[\begin{array}{cc}
z \mathbf{I} & -i \kappa \mathbf{I} \\
i \kappa \mathbf{I} & z^{-1} \mathbf{I}
\end{array}\right]
$$

where $z=\sqrt{\mu_{0} / \epsilon_{0}}>0$ is the impedance and $\kappa=\chi c_{0}$ is the chirality parameter. The four non-zero simple eigenvalues and their corresponding eigenvectors are

$$
\begin{array}{ll}
\mathbf{e}^{1} & \sim\left(\begin{array}{c}
-i \hat{\mathbf{p}}_{\perp}^{1}+\hat{\mathbf{p}}_{\perp}^{2} \\
-z^{-1} \hat{\mathbf{p}}_{\perp}^{1}-i z^{-1} \hat{\mathbf{p}}_{\perp}^{2}
\end{array}\right), \quad \Omega^{1}=c_{0}|\mathbf{p}|(1+\kappa)^{-1} ; \\
\mathbf{e}^{2} & \sim\left(\begin{array}{c}
i \hat{\mathbf{p}}_{\perp}^{1}+\hat{\mathbf{p}}_{\perp}^{2} \\
-z^{-1} \hat{\mathbf{p}}_{\perp}^{1}+i z^{-1} \hat{\mathbf{p}}_{\perp}^{2}
\end{array}\right), \quad \Omega^{2}=c_{0}|\mathbf{p}|(1-\kappa)^{-1} ; \\
\mathbf{e}^{3} & \sim\left(\begin{array}{c}
-i \hat{\mathbf{p}}_{\perp}^{1}+\hat{\mathbf{p}}_{\perp}^{2} \\
z^{-1} \hat{\mathbf{p}}_{\perp}^{1}+i z^{-1} \hat{\mathbf{p}}_{\perp}^{2}
\end{array}\right), \quad \Omega^{3}=c_{0}|\mathbf{p}|(\kappa-1)^{-1} ; \\
\mathbf{e}^{4} & \sim\left(\begin{array}{c}
i \hat{\mathbf{p}}_{\perp}^{1}+\hat{\mathbf{p}}_{\perp}^{2} \\
z^{-1} \hat{\mathbf{p}}_{\perp}^{1}-i z^{-1} \hat{\mathbf{p}}_{\perp}^{2}
\end{array}\right), \quad \Omega^{4}=c_{0}|\mathbf{p}|(-\kappa-1)^{-1} .
\end{array}
$$

Note also that $\Omega^{4}=-\Omega^{1}, \Omega^{3}=-\Omega^{2}$. As $|\kappa|<1, \mathbf{e}^{1}, \mathbf{e}^{2}$ are the forward propagating modes and $\mathbf{e}^{3}, \mathbf{e}^{4}$ the backward propagating modes.

7.3. Birefrigence in anisotropic crystals. The only optically isotropic crystal is the cubic crystal. In the system of principal dielectric axes, the permitivity-permeability tensor of a crystal, which is always a real, symmetric matrix, can be diagonalized as $\mathbf{K}_{0}=$ $\operatorname{diag}\left[\epsilon_{x}, \epsilon_{y}, \epsilon_{z}, 1,1,1\right]$. One type of anisotropic crystals are the uniaxial crystals for which $\epsilon_{x}=\epsilon_{y}=\epsilon_{\perp} \neq \epsilon_{z}=\epsilon_{\|}$(if the distinguished direction, the optic axis, is taken as the $z$-axis). There exist two distinct dispersion relations for the forward modes

$$
\Omega^{o}=\frac{|\mathbf{p}|}{\sqrt{\epsilon_{\perp}}}, \quad \Omega^{e}=\sqrt{\frac{p_{3}^{2}}{\epsilon_{\perp}}+\frac{p_{1}^{2}+p_{2}^{2}}{\epsilon_{\|}}} .
$$

The backward modes correspond to $-\Omega^{e},-\Omega^{o}$. The corresponding wavevector surface consists of a sphere and an ovaloid, a surface of revolution. The former corresponds to an ordinary wave with a velocity independent of the wavevector, the latter an extraordinary wave with a velocity depending on the angle between the wavevector and the optic axis [3].

Let $\mathbf{d}^{o}, \mathbf{d}^{e}$ be the associated eigenvectors. Set $\mathbf{K}_{0}^{\epsilon}=\operatorname{diag}\left[\epsilon_{\perp}, \epsilon_{\perp}, \epsilon_{\|}\right]$and let $\mathbf{a}^{\sigma}$ solve the following symmetric eigenvalue problem:

$$
-\mathbf{p} \times\left(\mathbf{K}_{0}^{\epsilon}\right)^{-1} \mathbf{p} \times \mathbf{a}^{\sigma}=\left(\Omega^{\sigma}\right)^{2} \mathbf{a}^{\sigma}, \quad \sigma=e, o .
$$

Then the eigenvectors $\mathbf{d}^{\sigma}$ can be written as

$$
\mathbf{d}^{\sigma} \sim\left(\begin{array}{c}
-\mathbf{p} \times \mathbf{a}^{\sigma} \\
\Omega^{\sigma} \mathbf{a}^{\sigma} \\
13
\end{array}\right), \quad \sigma=e, o .
$$


The same formula applies to the backward modes. Eq. (54) has the following solutions

$$
\mathbf{a}^{e}=\left(-p_{2}, p_{1}, 0\right)^{\dagger}, \quad \mathbf{a}^{o}=\left(p_{1}, p_{2},-\frac{p_{1}^{2}+p_{2}^{2}}{p_{3}}\right)^{\dagger}
$$

from which we deduce that the wave is linearly polarized.

7.4. Gyrotropic media: magneto-optical effect. For an isotrpic medium [11 in motion or in the presence of a static external magnetic field $\mathbf{H}_{\text {ext }}$ the permittivity tensor $\mathbf{K}_{0}^{\epsilon}$ is no longer symmetrical; it is generally a complex Hermitian matrix. Here we consider the simplest such constitutive relation

$$
\mathbf{D}=\epsilon_{0} \mathbf{E}-i \mathbf{g} \times \mathbf{E}, \quad \mathbf{B}=\mathbf{H}
$$

where $\mathbf{g}=f \mathbf{H}_{\text {ext }}, f \in \mathbb{R}$, is the gyration vector. Equivalently, we can write

$$
\mathbf{E}=\frac{1}{\epsilon_{0}^{2}-|\mathbf{g}|^{2}}\left(\epsilon_{0} \mathbf{D}+i \mathbf{g} \times \mathbf{D}-\frac{1}{\epsilon_{0}} \mathbf{g g}^{\dagger} \mathbf{D}\right) .
$$

In this case there are two distinct forward dispersion relations [1]

$$
\Omega^{1}=c_{0}\left|\mathbf{p}+\frac{\Omega^{1}}{2} \mathbf{g}\right|, \quad \Omega^{2}=c_{0}\left|\mathbf{p}-\frac{\Omega^{2}}{2} \mathbf{g}\right|
$$

where $c_{0}=1 / \sqrt{\epsilon_{0}}$. Clearly the wave-vector surface consists of two spheres of the same radius but different centers. This should be contrasted with the case of chiral media for which the wave-vector surface consists of two concentric spheres of different radii.

The associated eigenvectors $\mathbf{d}^{\sigma}, \sigma=1,2$ can be written as in (55) with $\mathbf{a}^{\sigma}$ solving (154) and with

$$
\mathbf{K}_{0}^{\epsilon}=\left[\begin{array}{ccc}
\epsilon_{0} & i g_{3} & -i g_{2} \\
-i g_{3} & \epsilon_{0} & i g_{1} \\
i g_{2} & -i g_{1} & \epsilon_{0}
\end{array}\right] .
$$

Let $\mathbf{g}=g_{1} \hat{\mathbf{p}}_{\perp}^{1}+g_{2} \hat{\mathbf{p}}_{\perp}^{2}+g_{3} \hat{\mathbf{p}}$. We can write the three-dimensional vector $\mathbf{a}^{\sigma}$ as $\mathbf{a}^{\sigma}=\hat{\mathbf{p}}_{\perp}^{1}+\gamma_{\sigma} \hat{\mathbf{p}}_{\perp}^{2}$ with

$$
\gamma_{\sigma}=\frac{g_{2}^{2}-g_{1}^{2}-(-1)^{\sigma} \sqrt{\left(g_{1}^{2}+g_{2}^{2}\right)^{2}+4 \epsilon_{0}^{2} g_{3}^{2}}}{2\left(g_{1} g_{2}-i \epsilon_{0} g_{3}\right)}, \quad \sigma=1,2 .
$$

We see that the wave is in general elliptically polarized or linearly polarized when $\mathbf{g}$ is orthogonal to the wavevector $\mathbf{p}$ and circularly polarized when $\mathbf{g}$ is parallel to $\mathbf{p}$. Again, the simplicity of the eigenvalues implies that depolarization is absent in the gyrotropic media.

\section{Conclusion}

The main contribution of this work is the derivation of the 2f-RT equations (45), (47) for the $2 \mathrm{f}-\mathrm{WD}$ in the weak-disorder regime based on the first Wigner-Moyal equation (7). All the terms in the equations can be explicitly calculated from the materials properties.

Let us turn to the second Wigner-Moyal equation (8) and briefly discuss its implications. By the same multi-scale expansion, the leading order term from eq. (8) is

$$
2 \bar{\omega} \overline{\mathbf{W}}=i p_{j} \mathbf{R}_{j} \mathbf{L} \overline{\mathbf{W}}+i \overline{\mathbf{W}} \mathbf{L} p_{j} \mathbf{R}_{j}
$$


which, along with (14), then implies that the wavenumber $\mathbf{p}$ should be restricted to the surface:

$$
\bar{\omega}=\Omega^{\sigma}(\mathbf{p}) .
$$

Denote the area element of the surface by $d \Omega$. Hence the mutual coherence in this regime is given approximately as

$$
\begin{aligned}
& \left\langle\mathbf{u}\left(\mathbf{x}_{1}, t_{1}\right) \mathbf{u}^{\dagger}\left(\mathbf{x}_{2}, t_{2}\right)\right\rangle \\
& \sim \sum_{\sigma, \alpha \zeta} \iint e^{-i \omega^{\prime} t} e^{-i \tau \bar{\omega} / \ell} \int_{\bar{\omega}=\Omega^{\sigma}(\mathbf{p})} e^{i \mathbf{p}^{\dagger}\left(\mathbf{x}_{1}-\mathbf{x}_{2}\right) / \ell} \bar{W}_{\alpha \zeta}^{\sigma}\left(\frac{\mathbf{x}_{1}+\mathbf{x}_{2}}{2}, \mathbf{p} ; \bar{\omega}, \omega^{\prime}\right) \mathbf{D}^{\sigma, \alpha \zeta}(\mathbf{p}, \mathbf{p}) d \Omega(\mathbf{p}) d \bar{\omega} d \omega^{\prime}
\end{aligned}
$$

where the coherence matrix $\overline{\mathbf{W}}^{\sigma}=\left[\bar{W}_{\alpha \zeta}^{\sigma}\right]$ satisfies the 2f-RT equations (45) and $d \Omega(\mathbf{p})$ is the area element of the surface $\bar{\omega}=\Omega^{\sigma}(\mathbf{p})$.

\section{REFERENCES}

[1] Yu. N. Barabanekov, A. G. Vinogradov, Yu. A. Kravtsov and V.I. Tartarski, "Application of the theory of multiple scattering of waves to the derivation of the radiation transfer equation for a statistically inhomogeneous medium," Radiophys. Quantum Electron. 15, 1420 - 1425 (1972).

[2] I. Bialynicki-Birula, "Photon wave function," Prog. Opt. 36, 245-294 (1996).

[3] M. Born and W. Wolf, Principles of Optics, 7-th (expanded) edition (Cambridge University Press, 1999).

[4] S. Chandrasekhar, Radiative Transfer (Dover Publications, New York, 1960).

[5] D. K. Cheng and 1. A. Kong, "Covariant descriptions of bianisotropic media," Proc. IEEE 56, pp. 248-251 (1968).

[6] A.C. Fannjiang, "Two-frequency radiative transfer and asymptotic solution," J. Opt. Soc. Am. 24 (2007), 2248-2256.

[7] A. C. Fannjiang, "Two-frequency radiative transfer: Maxwell equations in random dielectrics, " to appear J. Opt. Soc. Am. A (2007).

[8] R.L. Fante, "Relationship between radiative transport theory and Maxwell's equations in dielectric media," J. Opt. Soc. Am. 71, 460-468 (1981).

[9] J. H. Hannay, "Radiative transfer: exact Rayleigh scattering series and a formula for daylight," Proc. Roy. Soc. London 463, 2729-2751(2007)

[10] A. Kokhanovsky, Polarization Optics of Random Media (Springer, 2003).

[11] L.D. Landau, E.M. Lifshitz and L.P. Pitaevskii, Electrodynamics of Continuous Media (Elsevier Butterworth-Heinemann, Oxford, 1984)

[12] Lindell I V, Sihvola A H, Tretyakov S A and Viitanen A J, Electromagnetic Waves in Chiral and Bi-isotropic Media (Boston, MA: Artech House, 1994)

[13] C.W. Law and K. Watson, "Radiation transport along curved ray paths," J. Math. Phys. 11, 3125-3137 (1970).

[14] M. Mishchenko, L. Travis, A. Lacis, Multiple Scattering of Light by Particles: Radiative Transfer and Coherent Backscattering (Cambridge University Press, Cambridge, 2006).

[15] G. C. Papanicolaou and R. Burridge, "Transport equations for Stokes' parameters from Maxwell's equations in a random medium," J. Math. Phys. 16, 2074-2085 (1975).

[16] T.H. O'Dell, The Electrodynamics of Magneto-Electric Media (Amsterdam: North-Holland, 1970).

[17] L. Ryzhik, G. Papanicolaou and J.B. Keller, "Transport equations for elastic and other waves in random media," Wave Motion 24, 327-370 (1996).

[18] D. R. Smith and N. Kroll, "Negative refractive index in left-handed materials," Phys. Rev. Lett. 85, $2933(2000)$.

[19] D.R. Smith, J.B. Pendry and M.C.K. Wiltshire, "Metamaterials and negative refractive index," Science 305, 788-792 (2004). 


\section{Appendix A. Derivation of Wigner-Moyal equation}

From the Maxwell equations, we have

$$
\begin{aligned}
i \omega_{1} \mathbf{W}= & \frac{1}{(2 \pi)^{3}} \int e^{-i \mathbf{p}^{\dagger} \mathbf{y}} \mathbf{R}_{j} \partial_{x_{j}}\left(\mathbf{L}\left(\mathbf{x}+\frac{\ell \mathbf{y}}{2}\right) \mathbf{U}_{1}\right) \mathbf{U}_{2}^{\dagger} d \mathbf{y} \\
= & \frac{2 i}{\ell(2 \pi)^{3}} p_{j} \mathbf{R}_{j} \int e^{-i \mathbf{p}^{\dagger} \mathbf{y}} \mathbf{L}\left(\mathbf{x}+\frac{\ell \mathbf{y}}{2}\right) \mathbf{U}_{1} \mathbf{U}_{2}^{\dagger} d \mathbf{y} \\
& +\frac{1}{(2 \pi)^{3}} \int e^{-i \mathbf{p}^{\dagger} \mathbf{y}} \mathbf{R}_{j} \mathbf{L}\left(\mathbf{x}+\frac{\ell \mathbf{y}}{2}\right) \mathbf{U}_{1} \partial_{x_{j}} \mathbf{U}_{2}^{\dagger} d \mathbf{y}
\end{aligned}
$$

after changing variable and integrating by parts. Using the identity

$$
\begin{aligned}
\mathbf{R}_{j} \partial_{x_{j}} \int e^{-i \mathbf{p}^{\dagger} \mathbf{y}} \mathbf{L}\left(\mathbf{x}+\frac{\ell \mathbf{y}}{2}\right) \mathbf{U}_{1} \mathbf{U}_{2}^{\dagger} d \mathbf{y}= & \int e^{-i \mathbf{p}^{\dagger} \mathbf{y}} \mathbf{R}_{j} \partial_{x_{j}}\left[\mathbf{L}\left(\mathbf{x}+\frac{\ell \mathbf{y}}{2}\right) \mathbf{U}_{1}\right] \mathbf{U}_{2}^{\dagger} d \mathbf{y} \\
& +\int e^{-i \mathbf{p}^{\dagger} \mathbf{y}} \mathbf{R}_{j} \mathbf{L}\left(\mathbf{x}+\frac{\ell \mathbf{y}}{2}\right) \mathbf{U}_{1} \partial_{x_{j}} \mathbf{U}_{2}^{\dagger} d \mathbf{y}
\end{aligned}
$$

we then obtain

$$
\begin{aligned}
i \frac{\omega_{1}}{\ell} \mathbf{W}= & \frac{1}{(2 \pi)^{3}} \int e^{-i \mathbf{p}^{\dagger} \mathbf{y}} \mathbf{R}_{j} \partial_{x_{j}}\left(\mathbf{L}\left(\mathbf{x}+\frac{\ell \mathbf{y}}{2}\right) \mathbf{U}_{1}\right) \mathbf{U}_{2}^{\dagger} d \mathbf{y} \\
= & \frac{i}{\ell(2 \pi)^{3}} p_{j} \mathbf{R}_{j} \int e^{-i \mathbf{p}^{\dagger} \mathbf{y}} \mathbf{L}\left(\mathbf{x}+\frac{\ell \mathbf{y}}{2}\right) \mathbf{U}_{1} \mathbf{U}_{2}^{\dagger} d \mathbf{y} \\
& +\frac{1}{2(2 \pi)^{3}} \mathbf{R}_{j} \partial_{x_{j}} \int e^{-i \mathbf{p}^{\dagger} \mathbf{y}} \mathbf{L}\left(\mathbf{x}+\frac{\ell \mathbf{y}}{2}\right) \mathbf{U}_{1} \mathbf{U}_{2}^{\dagger} d \mathbf{y}
\end{aligned}
$$

Similarly,

$$
\begin{aligned}
-i \frac{\omega_{2}}{\ell} \mathbf{W}= & \frac{1}{(2 \pi)^{3}} \int e^{-i \mathbf{p}^{\dagger} \mathbf{y}} \mathbf{U}_{1} \nabla_{\mathbf{x}}\left(\mathbf{U}_{2}^{\dagger} \mathbf{L}\left(\mathbf{x}-\frac{\ell \mathbf{y}}{2}\right)\right) \mathbf{R}_{j} d \mathbf{y} \\
= & -\frac{i}{\ell(2 \pi)^{3}} \int e^{-i \mathbf{p}^{\dagger} \mathbf{y}} \mathbf{U}_{1} \mathbf{U}_{2}^{\dagger} \mathbf{L}\left(\mathbf{x}-\frac{\ell \mathbf{y}}{2}\right) d \mathbf{y} p_{j} \mathbf{R}_{j} \\
& +\frac{1}{2(2 \pi)^{3}} \nabla_{\mathbf{x}} \int e^{-i \mathbf{p}^{\dagger} \mathbf{y}} \mathbf{U}_{1} \mathbf{U}_{2}^{\dagger} \mathbf{L}\left(\mathbf{x}-\frac{\ell \mathbf{y}}{2}\right) d \mathbf{y} \mathbf{R}_{j}
\end{aligned}
$$

By the spectral representation of $\mathbf{L}$ we write

$$
\begin{aligned}
& \frac{1}{(2 \pi)^{3}} \int e^{-i \mathbf{p}^{\dagger} \mathbf{y}} \mathbf{L}\left(\mathbf{x}+\frac{\ell \mathbf{y}}{2}\right) \mathbf{U}_{1} \mathbf{U}_{2}^{\dagger} d \mathbf{y}=\int e^{i \mathbf{q}^{\dagger} \mathbf{x}} \widehat{\mathbf{L}}(\mathbf{q}) \mathbf{W}\left(\mathbf{p}-\frac{\ell \mathbf{q}}{2}\right) d \mathbf{q} \\
& \frac{1}{(2 \pi)^{3}} \int e^{-i \mathbf{p}^{\dagger} \mathbf{y}} \mathbf{U}_{1} \mathbf{U}_{2}^{\dagger} \mathbf{L}\left(\mathbf{x}-\frac{\ell \mathbf{y}}{2}\right) d \mathbf{y}=\int \mathbf{W}\left(\mathbf{p}+\frac{\ell \mathbf{q}}{2}\right) \widehat{\mathbf{L}}(\mathbf{q}) e^{i \mathbf{q}^{\dagger} \mathbf{x}} d \mathbf{q}
\end{aligned}
$$

Adding or subtracting (57) and (58) with (59)-(60) we obtain the Wigner-Moyal equations. 


\section{Appendix B. Derivation of geOMetrical optics EQUation}

Consider the following term from $\mathcal{P}_{2} \overline{\mathbf{W}}$ :

$$
\begin{aligned}
& \text { (61) } \operatorname{Tr}\left[\mathbf{E}^{\tau, \xi \nu \dagger} \mathbf{R}_{j} \partial_{x_{j}}\left[\mathbf{L} \bar{W}_{\alpha \zeta}^{\sigma} \mathbf{D}^{\sigma, \alpha \zeta}\right]\right] \\
& =\operatorname{Tr}\left[\mathbf{E}^{\tau, \xi \nu \dagger} \mathbf{R}_{j} \mathbf{L} \partial_{x_{j}}\left[\bar{W}_{\alpha \zeta}^{\sigma} \mathbf{D}^{\sigma, \alpha \zeta}\right]\right]+\operatorname{Tr}\left[\mathbf{E}^{\tau, \xi \nu \dagger} \mathbf{R}_{j} \partial_{x_{j}} \mathbf{L} \bar{W}_{\alpha \zeta}^{\sigma} \mathbf{D}^{\sigma, \alpha \zeta}\right] \\
& =\operatorname{Tr}\left[\mathbf{E}^{\tau, \xi \nu \dagger} \mathbf{R}_{j} \mathbf{L} \partial_{x_{j}} \bar{W}_{\alpha \zeta}^{\sigma} \mathbf{D}^{\sigma, \alpha \zeta}\right]+\operatorname{Tr}\left[\mathbf{E}^{\tau, \xi \nu \dagger} \mathbf{R}_{j} \mathbf{L} \bar{W}_{\alpha \zeta}^{\sigma} \partial_{x_{j}} \mathbf{D}^{\sigma, \alpha \zeta}\right]+\operatorname{Tr}\left[\mathbf{E}^{\tau, \xi \nu \dagger} \mathbf{R}_{j} \partial_{x_{j}} \mathbf{L} \bar{W}_{\alpha \zeta}^{\sigma} \mathbf{D}^{\sigma, \alpha \zeta}\right]
\end{aligned}
$$

The first term on the right hand side of (61) can be calculated as

$$
\begin{aligned}
& \operatorname{Tr}\left[\mathbf{E}^{\tau, \xi \nu \dagger} \mathbf{R}_{j} \mathbf{L} \cdot \partial_{x_{j}} \bar{W}_{\alpha \zeta}^{\sigma} \mathbf{D}^{\sigma, \alpha \zeta}\right] \\
= & \operatorname{Tr}\left[\mathbf{E}^{\tau, \xi \nu \dagger} \partial_{p_{j}}\left[p_{l} \mathbf{R}_{l} \mathbf{L}\right] \partial_{x_{j}} \bar{W}_{\alpha \zeta}^{\sigma} \mathbf{D}^{\sigma, \alpha \zeta}\right] \\
= & \operatorname{Tr}\left[\mathbf{e}^{\tau, \nu} \partial_{p_{j}}\left[\mathbf{e}^{\tau, \xi \dagger} p_{l} \mathbf{R}_{l} \mathbf{L}\right] \partial_{x_{j}} \bar{W}_{\alpha \zeta}^{\sigma} \mathbf{D}^{\sigma, \alpha \zeta}\right]-\operatorname{Tr}\left[\mathbf{e}^{\tau, \nu} \partial_{p_{j}}\left[\mathbf{e}^{\tau, \xi \dagger}\right] p_{l} \mathbf{R}_{l} \mathbf{L} \partial_{x_{j}} \bar{W}_{\alpha \zeta}^{\sigma} \mathbf{D}^{\sigma, \alpha \zeta}\right] \\
= & \operatorname{Tr}\left[\mathbf{E}^{\tau, \xi \nu \dagger} \partial_{p_{j}} \Omega^{\tau} \partial_{x_{j}} \bar{W}_{\alpha \zeta}^{\sigma} \mathbf{D}^{\sigma, \alpha \zeta}\right]+\operatorname{Tr}\left[\mathbf{e}^{\tau, \nu} \partial_{p_{j}}\left[\mathbf{e}^{\tau, \xi \dagger}\right]\left[\Omega^{\tau}-p_{l} \mathbf{R}_{l} \mathbf{L}\right] \partial_{x_{j}} \bar{W}_{\alpha \zeta}^{\sigma} \mathbf{D}^{\sigma, \alpha \zeta}\right] \\
= & \delta_{\tau \delta} \delta_{\xi \alpha} \delta_{\nu \zeta} \nabla_{\mathbf{p}} \Omega^{\tau} \cdot \nabla_{\mathbf{x}} \bar{W}_{\xi \nu}^{\tau}
\end{aligned}
$$

using the eigenvector property and (10) while the last term on the right hand side of (61) is

$$
\operatorname{Tr}\left[\mathbf{E}^{\tau, \xi \nu \dagger} \mathbf{R}_{j} \partial_{x_{j}} \mathbf{L} \bar{W}_{\alpha \zeta}^{\sigma} \mathbf{D}^{\sigma, \alpha \zeta}\right]=\delta_{\tau \sigma} \delta_{\nu \zeta} \mathbf{e}^{\tau, \xi \dagger} \mathbf{R}_{j} \partial_{x_{j}} \mathbf{L d}^{\tau, \alpha} \bar{W}_{\alpha \nu}^{\tau} .
$$

We turn to the middle term on the right hand side of (61). We have the following calculation.

$$
\begin{aligned}
& \sum_{\sigma, \alpha, \zeta} \operatorname{Tr}\left[\mathbf{E}^{\tau, \xi \nu \dagger} \mathbf{R}_{j} \mathbf{L} \bar{W}_{\alpha \zeta}^{\sigma}\left[\partial_{x_{j}} \mathbf{d}^{\sigma, \alpha} \mathbf{d}^{\sigma, \zeta \dagger}+\mathbf{d}^{\sigma, \alpha} \partial_{x_{j}} \mathbf{d}^{\sigma, \zeta \dagger}\right]\right] \\
= & \mathbf{e}^{\tau, \xi \dagger} \mathbf{R}_{j} \mathbf{L} \partial_{x_{j}} \mathbf{d}^{\tau, \alpha} \bar{W}_{\alpha \nu}^{\tau}+\sum_{\sigma, \alpha, \zeta} \operatorname{Tr}\left[\mathbf{e}^{\tau, \nu} \partial_{p_{l}}\left[\mathbf{e}^{\tau, \xi \dagger} p_{j} \mathbf{R}_{j} \mathbf{L}\right] \bar{W}_{\alpha \zeta}^{\sigma} \mathbf{d}^{\sigma, \alpha} \partial_{x_{l}} \mathbf{d}^{\sigma, \zeta \dagger}\right] \\
& -\sum_{\sigma, \alpha, \zeta} \operatorname{Tr}\left[\mathbf{e}^{\tau, \nu} \partial_{p_{l}} \mathbf{e}^{\tau, \xi \dagger} \bar{W}_{\alpha \zeta}^{\sigma} p_{j} \mathbf{R}_{j} \mathbf{L} \mathbf{d}^{\sigma, \alpha} \partial_{x_{l}} \mathbf{d}^{\sigma, \zeta \dagger}\right] \\
= & \mathbf{e}^{\tau, \xi \dagger} \mathbf{R}_{j} \mathbf{L} \partial_{x_{j}} \mathbf{d}^{\tau, \alpha} \bar{W}_{\alpha \nu}^{\tau}+\sum_{\sigma, \alpha, \zeta} \operatorname{Tr}\left[\mathbf{e}^{\tau, \nu} \partial_{p_{l}}\left[\Omega^{\tau} \mathbf{e}^{\tau, \xi \dagger}\right] \bar{W}_{\alpha \zeta}^{\sigma} \mathbf{d}^{\sigma, \alpha} \partial_{x_{l}} \mathbf{d}^{\sigma, \zeta \dagger}\right] \\
& -\sum_{\sigma, \alpha, \zeta} \operatorname{Tr}\left[\mathbf{e}^{\tau, \nu} \partial_{p_{l}} \mathbf{e}^{\tau, \xi \dagger} \bar{W}_{\alpha \zeta}^{\sigma} \Omega^{\sigma} \mathbf{d}^{\sigma, \alpha} \partial_{x_{l}} \mathbf{d}^{\sigma, \zeta \dagger}\right] \\
= & \sum_{\alpha} \mathbf{e}^{\tau, \xi \dagger} \mathbf{R}_{j} \mathbf{L} \partial_{x_{j}} \mathbf{d}^{\tau, \alpha} \bar{W}_{\alpha \nu}^{\tau}+\sum_{\zeta} \partial_{p_{j}} \Omega^{\tau} \partial_{x_{j}} \mathbf{d}^{\tau, \zeta \dagger} \mathbf{e}^{\tau, \nu} \bar{W}_{\xi \zeta}^{\tau} \\
& +\sum_{\sigma, \alpha, \zeta}\left(\Omega^{\tau}-\Omega^{\sigma}\right) \bar{W}_{\alpha \zeta}^{\sigma} \partial_{p_{j}} \mathbf{e}^{\tau, \xi \dagger} \mathbf{d}^{\sigma, \alpha} \partial_{x_{j}} \mathbf{d}^{\sigma, \zeta \dagger} \mathbf{e}^{\tau, \nu} .
\end{aligned}
$$

The second part in $\mathcal{P}_{2} \mathbf{\mathbf { W }}$ can be calculated in the same manner. The counterpart of (62) yields exactly the same expression as the right hand side of (62) while the counterparts of (63) and (64) yield, respectively

$$
\sum_{\zeta} \bar{W}_{\xi \zeta}^{\tau} \mathbf{d}^{\tau, \zeta \dagger} \partial_{x_{j}} \mathbf{L R}_{j} \mathbf{e}^{\tau, \nu}
$$


and

$$
\begin{aligned}
& \sum_{\sigma, \alpha, \zeta} \operatorname{Tr}\left[\mathbf{E}^{\tau, \xi \nu \dagger} \bar{W}_{\alpha \zeta}^{\sigma}\left[\partial_{x_{j}} \mathbf{d}^{\sigma, \alpha} \mathbf{d}^{\sigma, \zeta}+\mathbf{d}^{\sigma, \alpha} \partial_{x_{j}} \mathbf{d}^{\sigma, \zeta}\right] \mathbf{L} \mathbf{R}_{j}\right] \\
= & \sum_{\zeta} \bar{W}_{\xi \zeta}^{\tau} \partial_{x_{j}} \mathbf{d}^{\tau, \zeta \dagger} \mathbf{L} \mathbf{R}_{j} \mathbf{e}^{\tau, \nu}+\sum_{\alpha} \mathbf{e}^{\tau, \xi \dagger} \partial_{x_{l}} \mathbf{d}^{\tau, \alpha} \partial_{p_{l}} \Omega^{\tau} \bar{W}_{\alpha \nu}^{\tau} \\
& +\sum_{\sigma, \alpha, \zeta}\left(\Omega^{\tau}-\Omega^{\sigma}\right) \bar{W}_{\alpha \zeta}^{\sigma} \mathbf{e}^{\tau, \xi \dagger} \partial_{x_{l}} \mathbf{d}^{\sigma, \alpha} \mathbf{d}^{\sigma, \zeta \dagger} \partial_{p_{l}} \mathbf{e}^{\tau, \nu}
\end{aligned}
$$

Next let us turn to the first part in $\mathcal{P}_{1} \overline{\mathbf{W}}$ :

$$
\begin{aligned}
& \sum_{\sigma, \alpha, \zeta} \operatorname{Tr}\left[\mathbf{E}^{\tau, \xi \nu \dagger} p_{j} \mathbf{R}_{j} \nabla_{\mathbf{x}} \mathbf{L} \cdot \nabla_{\mathbf{p}}\left[\bar{W}_{\alpha \zeta}^{\sigma} \mathbf{d}^{\sigma, \alpha} \mathbf{d}^{\sigma, \zeta \dagger}\right]\right] \\
= & \sum_{\alpha} \mathbf{e}^{\tau, \xi \dagger} p_{j} \mathbf{R}_{j} \nabla_{\mathbf{x}} \mathbf{L} \cdot \nabla_{\mathbf{p}} \bar{W}_{\alpha \nu}^{\tau} \mathbf{d}^{\tau, \alpha}+\sum_{\sigma, \alpha, \zeta} \operatorname{Tr}\left[\mathbf{E}^{\tau, \xi \nu \dagger} p_{j} \mathbf{R}_{j} \partial_{x_{j}} \mathbf{L} \bar{W}_{\alpha \zeta}^{\sigma} \partial_{p_{j}}\left[\mathbf{d}^{\sigma, \alpha} \mathbf{d}^{\sigma, \zeta \dagger}\right]\right] .
\end{aligned}
$$

The first term on the right hand side of (66) equals

$$
\sum_{\alpha} \partial_{x_{j}}\left(\Omega^{\tau} \mathbf{e}^{\tau, \xi \dagger}\right) \mathbf{d}^{\tau, \alpha} \partial_{p_{j}} \bar{W}_{\alpha \nu}^{\tau}-\sum_{\alpha} \partial_{x_{j}} \mathbf{e}^{\tau, \xi \dagger} p_{j} \mathbf{R}_{j} \mathbf{L d}^{\tau, \alpha} \partial_{p_{j}} \bar{W}_{\alpha \nu}^{\tau}=\nabla_{\mathbf{x}} \Omega^{\tau} \cdot \nabla_{\mathbf{p}} \bar{W}_{\xi \nu}^{\tau}
$$

while the second term can be calculated as

$$
\begin{aligned}
& \sum_{\sigma, \alpha, \zeta} \partial_{x_{j}}\left(\Omega^{\tau} \mathbf{e}^{\tau, \xi \dagger}\right) \bar{W}_{\alpha \zeta}^{\sigma} \partial_{p_{j}}\left[\mathbf{d}^{\sigma, \alpha} \mathbf{d}^{\sigma, \zeta \dagger}\right] \mathbf{e}^{\tau, \nu}-\sum_{\sigma, \alpha, \zeta} \partial_{x_{j}} \mathbf{e}^{\tau, \xi \dagger} p_{j} \mathbf{R}_{j} \mathbf{L} \bar{W}_{\alpha \zeta}^{\sigma} \partial_{p_{j}}\left[\mathbf{d}^{\sigma, \alpha} \mathbf{d}^{\sigma, \zeta \dagger}\right] \mathbf{e}^{\tau, \nu} \\
= & \sum_{\alpha} \mathbf{e}^{\tau, \xi \dagger} \partial_{p_{j}} \mathbf{d}^{\tau, \alpha} \partial_{x_{j}} \Omega^{\tau} \bar{W}_{\alpha \nu}^{\tau}+\sum_{\zeta} \bar{W}_{\xi \zeta}^{\tau} \partial_{x_{j}} \Omega^{\tau} \partial_{p_{j}} \mathbf{d}^{\tau, \zeta \dagger} \mathbf{e}^{\tau, \nu}+\sum_{\alpha} \Omega^{\tau} \bar{W}_{\alpha \nu}^{\tau} \partial_{x_{j}} \mathbf{e}^{\tau, \xi \dagger} \partial_{p_{j}} \mathbf{d}^{\tau, \alpha} \\
& +\sum_{\sigma, \alpha, \zeta}\left(\Omega^{\tau}-\Omega^{\sigma}\right) \bar{W}_{\alpha \zeta}^{\sigma} \partial_{x_{j}} \mathbf{e}^{\tau, \xi \dagger} \mathbf{d}^{\sigma, \alpha} \partial_{p_{j}} \mathbf{d}^{\sigma, \zeta \dagger} \mathbf{e}^{\tau, \nu}-\sum_{\alpha} \bar{W}_{\alpha \nu}^{\tau} \partial_{x_{j}} \mathbf{e}^{\tau, \xi \dagger} p_{j} \mathbf{R}_{j} \mathbf{L} \partial_{p_{j}} \mathbf{d}^{\tau, \alpha}
\end{aligned}
$$

The last term on the right hand side of (67) can be further expressed as

$$
\begin{aligned}
& -\sum_{\alpha} \bar{W}_{\alpha \nu}^{\tau} \partial_{x_{j}} \mathbf{e}^{\tau, \xi \dagger} \partial_{p_{j}}\left[p_{j} \mathbf{R}_{j} \mathbf{L} \mathbf{d}^{\tau, \alpha}\right]+\sum_{\alpha} \bar{W}_{\alpha \nu}^{\tau} \partial_{x_{j}} \mathbf{e}^{\tau, \xi \dagger} \mathbf{R}_{j} \mathbf{L d}^{\tau, \alpha} \\
= & -\sum_{\alpha} \bar{W}_{\alpha \nu}^{\tau} \partial_{p_{j}} \Omega^{\tau} \partial_{x_{j}} \mathbf{e}^{\tau, \xi \dagger} \mathbf{d}^{\tau, \alpha}-\sum_{\alpha} \bar{W}_{\alpha \nu}^{\tau} \Omega^{\tau} \partial_{x_{j}} \mathbf{e}^{\tau, \xi \dagger} \partial_{p_{j}} \mathbf{d}^{\tau, \alpha} \\
& +\sum_{\alpha} \partial_{x_{j}} \mathbf{e}^{\tau, \xi \dagger} \mathbf{R}_{j} \mathbf{L} \mathbf{d}^{\tau, \alpha} \bar{W}_{\alpha \nu}^{\tau} .
\end{aligned}
$$

For the second part in $\mathcal{P}_{1} \mathbf{W}$, the counterpart of (67) yields exactly the same expression as (67) while the counterpart of (67) yields

$$
\begin{aligned}
& \sum_{\alpha} \mathbf{e}^{\tau, \xi \dagger} \partial_{p_{j}} \mathbf{d}^{\tau, \alpha} \partial_{x_{j}} \Omega^{\tau} \bar{W}_{\alpha \nu}^{\tau}+\sum_{\zeta} \bar{W}_{\xi \zeta}^{\tau} \partial_{x_{j}} \Omega^{\tau} \partial_{p_{j}} \mathbf{d}^{\tau, \zeta \dagger} \mathbf{e}^{\tau, \nu} \\
& +\sum_{\sigma, \alpha, \zeta}\left(\Omega^{\tau}-\Omega^{\sigma}\right) \bar{W}_{\alpha \zeta}^{\sigma} \mathbf{e}^{\tau, \xi \dagger} \partial_{p_{j}} \mathbf{d}^{\sigma, \alpha} \mathbf{d}^{\sigma, \zeta \dagger} \partial_{x_{j}} \mathbf{e}^{\tau, \nu}+\bar{W}_{\xi \zeta}^{\tau} \mathbf{d}^{\tau, \zeta \dagger} \mathbf{L} \mathbf{R}_{j} \partial_{x_{j}} \mathbf{e}^{\tau, \nu}-\bar{W}_{\xi \zeta}^{\tau} \partial_{p_{j}} \Omega^{\tau} \mathbf{d}^{\tau, \xi \dagger} \partial_{x_{j}} \mathbf{e}^{\tau, \nu}
\end{aligned}
$$

In the final expression, many of the above terms in $\mathcal{P}_{1} \overline{\mathbf{W}}-\mathcal{P}_{2} \overline{\mathbf{W}}$ cancel. For instance, all the terms involving $\left(\Omega^{\tau}-\Omega^{\sigma}\right)$, all the terms involving $\bar{W}_{\xi \zeta}^{\tau} \partial_{p_{j}} \Omega^{\tau}, \bar{W}_{\alpha \nu}^{\tau} \partial_{p_{j}} \Omega^{\tau}$ and all the terms 
involving $\Omega^{\tau} \bar{W}_{\xi \zeta}^{\tau}, \Omega^{\tau} \bar{W}_{\alpha \nu}^{\tau}$ cancel. Using the fact $\mathbf{e}^{\tau, \alpha}=\mathbf{L d}^{\tau, \alpha}$ and some algebra we obtain

$$
\begin{aligned}
& \operatorname{Tr}\left[\mathbf{E}^{\tau, \xi \nu \dagger} \mathcal{P}_{1} \overline{\mathbf{W}}\right]-\operatorname{Tr}\left[\mathbf{E}^{\tau, \xi \nu \dagger} \mathcal{P}_{2} \overline{\mathbf{W}}\right]-\nabla_{\mathbf{x}} \Omega^{\tau} \cdot \nabla_{\mathbf{p}} \bar{W}_{\xi \nu}^{\tau}+\nabla_{\mathbf{p}} \Omega^{\tau} \cdot \nabla_{\mathbf{x}} \bar{W}_{\xi \nu}^{\tau} \\
= & \sum_{\alpha} \mathbf{e}^{\tau, \xi \dagger} \partial_{p_{j}} \mathbf{d}^{\tau, \alpha} \partial_{x_{j}} \Omega^{\tau} \bar{W}_{\alpha \nu}^{\tau}+\sum_{\zeta} \bar{W}_{\xi \zeta}^{\tau} \partial_{x_{j}} \Omega^{\tau} \partial_{p_{j}} \mathbf{d}^{\tau, \zeta \dagger} \mathbf{e}^{\tau, \nu}+\frac{1}{2} \sum_{\alpha} \partial_{x_{j}} \mathbf{e}^{\tau, \xi \dagger} \mathbf{R}_{j} \mathbf{e}^{\tau, \alpha} \bar{W}_{\alpha \nu}^{\tau} \\
& -\frac{1}{2} \sum_{\alpha} \mathbf{e}^{\tau, \xi \dagger} \mathbf{R}_{j} \partial_{x_{j}} \mathbf{e}^{\tau, \alpha} \bar{W}_{\alpha \nu}^{\tau}+\frac{1}{2} \sum_{\zeta} \bar{W}_{\xi \zeta}^{\tau} \mathbf{e}^{\tau, \zeta \dagger} \mathbf{R}_{j} \partial_{x_{j}} \mathbf{e}^{\tau, \nu}-\frac{1}{2} \sum_{\zeta} \bar{W}_{\xi \zeta}^{\tau} \partial_{x_{j}} \mathbf{e}^{\tau, \zeta \dagger} \mathbf{R}_{j} \mathbf{e}^{\tau, \nu} .
\end{aligned}
$$

